Notre Dame Journal of Formal Logic

Volume XIV, Number 1, January 1973

NDJFAM

\title{
AXIOMATIC INSCRIPTIONAL SYNTAX \\ PART II: THE SYNTAX OF PROTOTHETIC
}

\section{FREDERICK RICKEY}

PART I* of this paper presented an axiomatized theory of general syntax, defined the usual notions of general syntax, and proved some typical theorems of general syntax. We claimed that this system is strong enough to precisely state the formative and deductive rules of formal languages. In PART II we support this claim by formulating, in a very precise way, the rule of procedure of Leśniewski's Protothetic. Chapter III is an informal introduction to Protothetic and its rule of procedure. Our metalogical system is extended there to a theory MP which is concerned explicitly with the syntax of Protothetic. This chapter is intended to motivate the Terminological Explanations of Chapter IV which culminate in the statement of the rule of Protothetic. In Chapter $V$ we sketch a proof that our formulation of the rule is equivalent to that given by Leśniewski in [18]. This proof shows that our system is strong enough to conduct certain metalogical investigations.

There are several reasons why we chose Protothetic as a test case for our syntactical system: 1) Since the rule of Protothetic is as complicated as the deductive rule for any theory it provides a good test of the usefulness of our system for formalizing theories. 2) Most papers on Protothetic only discuss the deductive rule from an informal point of view. It is hoped that our formulation of the rule, which is simpler than that given by Leśniewski [18], will serve to make this aspect of Leśniewski's work more accessible. 3) It was necessary, in order to make the comparisons that we do in Chapter $V$, to use a theory whose deductive rule had previously been precisely stated. Protothetic is one of the few such theories whose rule had been formulated without making excessive use of ordinary language, set theory, or inductive techniques. And, as we stated in PART I, it is our conviction that the formalization of a system should be done using the weakest possible metalogic.

*PART I of this paper appeared in the Notre Dame Journal of Formal Logic, vol. XIII (1972), pp. 1-33. The author would like to thank Professor Bolesław Sobociński for considerable advice. 


\section{CHAPTER III}

1 History and Informal Description of Protothetic. In 1923 Stanisław Leśniewski developed a bivalued system of logic which he called Protothetic. Besides including the full propositional calculus Protothetic contains variables of all semantical categories which can be generated from propositions. To explain, let us say that Protothetic is a type theory whose lowest type is that of propositions:

$$
p, q, r, \ldots .
$$

These can be combined, using unary, binary, ...., functors, to form new propositions:

$$
f(p), g(p q), h(p q r), \ldots .
$$

Then we can form functors which take previously introduced types as variables, for example:

$$
\varphi[f p], \psi<f g h>, \ldots .
$$

This process is continued indefinitely. After a constant functor with a given number of arguments is introduced we can use variables of that type. This will be explained more fully in section 3 .

Protothetic can be based on the law of simplification

$$
[p q]: p \supset . q \supset p
$$

together with the principal of bivalency for each semantical category. These are theses of the form

(2) $[f p]: . f(0) . \supset: f(1) . \supset . f(p)$

(3) $[F f]: \because: F<\mathrm{N}>. \supset:: F<\mathrm{FI}>. \supset: . F<\mathrm{Vr}>. \supset: F<\mathrm{As}>. \supset . F<f>$

There are, however, very grave difficulties with stating the rule of bivalency, for we must specify all the constants of a given semantical category before we can state the law of bivalency for that semantical category. This is not just a problem of specifying symbols for each of the constants, rather we must also state how they are defined or at least know that once the proper number have been defined that they are distinct. As this problem is still unsolved we use a different approach.

Another way to formulate Protothetic is to accept simplification (1), bivalency for propositions (2) and extensionality for every other semantical category.

The simplest form of extensionality is that for propositions

$$
[p q]: . p \equiv q . \equiv:[f]: f(p) . \equiv f(q),
$$

but this follows from (1) and (2). The theses of extensionality which are essential are those for all of the other semantical categories. These theses are of the form:

$$
\begin{aligned}
& \text { (5) }[f g]::[p]: f(p) . \equiv g(p): \equiv:[F]: F<f>\equiv . F<g> \\
& (6) \quad[f g]: .[p q]: f(p q) . \equiv . g(p q): \equiv:[F]: F<f>. \equiv . F<g>
\end{aligned}
$$


Since we have a method of formulating the rule of extensionality ${ }^{1}$ the difficulty is overcome.

(5) and (6) are stated using only the constants of equivalence. (1) and (2), however, use implication. It is highly desirable to base Protothetic on a single primitive term. Tarski [49] showed that conjunction could be defined in terms of equivalence and quantification ${ }^{2}$ :

$$
[p q]:: p . q . \equiv: .[f]: . p . \equiv: f(p) . \equiv . f(q)
$$

Since negation is easily defined:

$$
[p]: \sim p . \equiv: p . \equiv .[u] . u
$$

we have all the connectives needed for the propositional calculus. This allows Protothetic to be based on equivalence (as sole primitive). This has the added advantage that definitions can be stated in the most natural form, ${ }^{3}$ i.e., as equivalences:

(8) $\alpha \equiv \beta$

The successive simplification of the axiomatization of Protothetic was a long and complicated process. The details of this work and their history can be found in Leśniewski [18], [21], and Sobociński [48]. The system of Protothetic which we will be concerned with is based on Sobociński's 1945 axiom:

$$
\left.\left.\left.\left.{ }_{\llcorner} p q\right\lrcorner \gamma\left(\phi(p q)_{\llcorner} f_{\lrcorner}\left\ulcorner\phi\left(f\left(p f\left(p_{\llcorner} u\right\lrcorner u^{\urcorner}\right)\right)_{\llcorner} r\right\lrcorner{ }^{\prime} \phi(f(q r) \phi(q p))\right\urcorner\right)^{\urcorner}\right)\right\urcorner
$$

or, in the more familiar Peano-Russellian notation:

$$
[p q]:: p \equiv q . \equiv: .[f]: . f(p f(p 0)) . \equiv:[r]: f(q r) . \equiv q \equiv p
$$

This axiom is a dead object without some rules for deducing its consequences. We hinted above that we shall have rules of extensionality and definition. In addition we shall need rules of detachment, substitution, and a rule for distributing quantifiers.

The precise statement of these rules occupies the whole of Chapter IV. In order to make the Terminological Explanations of that chapter understandable we wish first to give an informal statement of the rule. But first we must discuss semantical categories.

2 Semantical Categories. In the axiom of Protothetic $[1,(9)]$ the basic unit is the biconditional ${ }^{4}$

$$
\phi(p q)
$$

or in the Peano-Russellian notation

$$
p \equiv q \text {. }
$$

Intuitively, equivalence is a functor which forms a proposition out of the two propositions which are its arguments. If we denote the semantical category of propositions by $S$ then, using the notation of Ajdukiewicz [1], we denote the semantical category of $\phi$ by 
In general the symbol

$$
\mathrm{C} / \mathrm{C}_{1} \mathrm{C}_{2} \ldots \mathrm{C}_{\mathrm{n}}
$$

represents the semantical category of a functor with $\mathrm{n}$ arguments of categories $\mathrm{C}_{1}, \mathrm{C}_{2}, \ldots, \mathrm{C}_{\mathrm{n}}$ respectively, which forms something of category C. For example, in the category $S / S$ the only constant functors are the four unary predicates $\mathrm{Vr}, \mathrm{Fl}, \mathrm{As}, \mathrm{N}$; in the category $\mathrm{S} / \mathrm{SS}$ we have the 16 binary functors. Semantically we want two functors to be in the same semantical category if they can be interchanged in all contexts without loss of meaning. ${ }^{5}$ So for example, the functors in

$$
\psi[f p], \varphi[f p]
$$

should belong to the same category, but we cannot decide to which until the categories of $f$ and $p$ are known. Similarily in

$$
[f]: f(p) . \equiv . *<>
$$

we want the two $f$ 's to be in the same category, but we cannot say which one until we know the category of $p$ or *. All that we can say is that $f$ belongs to the category $\mathrm{S} / \mathrm{C}$ and * to the category $\mathrm{S} /(\mathrm{S} / \mathrm{C})$, where $\mathrm{C}$ is the category of $p$.

Up to this point intersubstitutability has been the criteria for placing two functors in the same semantical category. This method will not suffice for our syntactical investigations for it uses the semantical notion of meaning.

Leśniewski was able to define semantical categories syntactically by letting the shape of the parentheses in which arguments are enclosed reveal the semantical categories of the arguments and their functors. He used the parentheses (--) to enclose two sentential variables. So when we see

$$
\phi-(p q)
$$

we know $\phi$ must be of the type S/SS since the parentheses tell us $p$ and $q$ are both in the category $S$ and that the functor is sentential.

For the purpose of formalizing Protothetic we cannot use any semantical notions. Thus it is absolutely necessary to replace the semantical notion of a semantical category by some syntactical notion.

Following the methods of Leśniewski we shall let the parentheses reveal the semantical categories of the arguments and functors involved. We admit that the motivation is completely semantical, but still, our presentation must be completely syntactical. Accordingly we shall speak in the future only of syntactical categories. We are using the term "syntactical category" instead of "semantical category" at the suggestion of Chomsky [6] and Bar-Hillel [45]. This notion is defined in Chapter IV by purely syntactical means. But since our motivation is semantical it agrees with the semantical categories presented above.

Because it is necessary, if one wishes to understand what follows, to have a tentative notion of syntactical category we present the following "definition": 
We shall say that two functors are in the same syntactical category if they have the same number of arguments, corresponding arguments are of the same syntactical categories, and the functors together with their arguments form objects of the same syntactical category.

3 Informal Statement of the Rule of Protothetic. As mentioned previously, the rule of procedure of Protothetic consists of five parts, viz., detachment, distribution of quantifiers, extensionality, substitution and definition. Let us consider them individually.

Detachment. If, at a given stage of development, Protothetic contains the thesis

$$
\oint(A B)
$$

and also the thesis

then we can add to Protothetic a thesis equiform to $B$. This rule is well enough known from other systems but we should remark that in (1) there is no main general quantifier preceding the biconditional. If we had

$$
\left.{ }_{\llcorner} p q \ldots\right\lrcorner \gamma(A(p q \ldots) B(p q \ldots))^{\top}
$$

as a thesis and also

$$
\left.{ }_{\llcorner} p q \ldots\right\lrcorner\ulcorner A(p q \ldots)\urcorner
$$

then we would have to distribute the quantifier in (3) before detaching.

Distribution of Quantifiers. If Protothetic contains a thesis (3) then we can distribute the quantifiers and get

$$
\oint\left({ }_{\llcorner} p q \ldots\right\lrcorner\left\ulcorner A(p q \ldots)^{\urcorner} p q \ldots{ }_{\llcorner} p(p q \ldots)^{\urcorner}\right)
$$

The rule is even stronger than this. It is stated so that we can distribute not only all, but any of the variables in the main general quantifier (MGQ) of (3). Thus we can also get

(6) $\left.\left.\quad \iota_{\llcorner} q \ldots\right\lrcorner\left\ulcorner_{(\llcorner} p\right\lrcorner\left\ulcorner A(p q \ldots)^{\urcorner}{ }_{\llcorner} p\right\lrcorner B(p q \ldots)^{\urcorner}\right)^{\urcorner}$

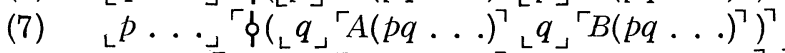

(8) $\llcorner\ldots\lrcorner\left\ulcorner\phi\left({ }_{\llcorner} p q\right\lrcorner\left\ulcorner_{A}(p q \ldots)_{\llcorner} p q\right\lrcorner\left\ulcorner B(p q \ldots)^{\urcorner}\right)^{\urcorner}\right.$

Protothetic also makes no use of vacuous quantifiers so if $A$ or $B$ contains no free occurrence of $p$ then $p$ is omitted from the quantifier immediately preceding $A$ or $B$ after distribution of $p$. For example from

$$
{ }_{L} p q r_{\lrcorner}\left\ulcorner\phi\left({ }_{L} p\right\lrcorner \operatorname{Vr}(p){ }_{L} s_{\lrcorner}\left\ulcorner\psi(p q r s)^{\urcorner}\right)\right\urcorner
$$

we can get, among others,

$$
\begin{aligned}
& \left.{ }_{\llcorner} q r\right\lrcorner \gamma_{\zeta}\left({ }_{L} p\right\lrcorner V_{\lrcorner}(p){ }_{L}{ }_{L} p s_{\lrcorner}\left\ulcorner\psi(p q r s)^{\urcorner}\right)^{\urcorner} \\
& \phi\left({ }_{L} p,\left\ulcorner\operatorname{Vr}(p)^{\urcorner}{ }_{\llcorner} p q r s,\left\ulcorner\psi(p q r s)^{\urcorner}\right)\right.\right.
\end{aligned}
$$

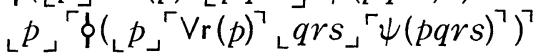

Notice that the stronger statement of the rule of distribution permits the simpler statement of the rule of detachment. 
The rule of quantification, as stated above, is strong enough so that we can derive the usual rules of quantification such as distribution of quantifiers through implication.

Extensionality. If the semantical categories

$$
\mathrm{S} / \mathrm{C}_{1} \mathrm{C}_{2} \ldots \mathrm{C}_{\mathrm{n}}, \mathrm{S} /\left(\mathrm{S} / \mathrm{C}_{1} \mathrm{C}_{2} \ldots \mathrm{C}_{\mathrm{n}}\right)
$$

have already been introduced into Protothetic by way of definition (see below) then the rule of extensionality allows us to add the following as a new thesis of Protothetic:

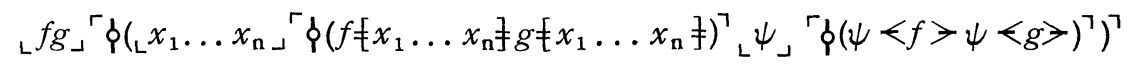

where the variables $x_{1}, \ldots, x_{\mathrm{n}}$ are of categories $C_{1}, \ldots, C_{n}$. To give a concrete example we state the law of extensionality for binary functors:

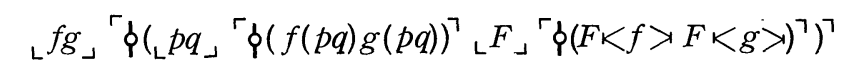

The rule does not allow us to add extensionality for the semantical category of propositions, since it is derivable from the axiom of Protothetic ( $C f$. Sobociński [48]).

The rule of extensionality allows us to add a thesis of extensionality for each semantical category (except for propositions), and not just those indicated above. We can also add extensionality when functors involving parameters (the so-called many link functors) are involved, e.g.,

$$
\left.\left.\left.\llcorner f g\lrcorner \Gamma_{\zeta}\left(L_{L} p q\right\lrcorner \Gamma \phi(f+p+(q) g+p+(q)){ }_{\llcorner} F\right\lrcorner\ulcorner\phi(F \propto f>F \propto g>0)\urcorner\right)\right\urcorner
$$

Substitution. The notion of substitution is well enough known from other systems of logic to need little comment here. The most important thing about substitution in Protothetic is that we never use incomplete symbols. ${ }^{6}$ In other systems of logic if one had

$$
\left.{ }_{L} f p\right\lrcorner \Gamma(f(p))^{\top}
$$

and wanted to get

$$
\left.{ }_{\llcorner} p\right\lrcorner\ulcorner\Phi(\phi(p p))\urcorner
$$

he would just say "substitute $\phi(p-)$ for $f$." On semantical grounds this is absurd, for $f$ and $\phi$ clearly belong to different semantical categories; so we certainly cannot substitute $\hat{\phi}$ for $f$. In Protothetic this substitution is accomplished via a definition:

$$
{ }_{L} p\ulcorner\phi(\phi(p p) \vee \mathrm{r}(p))\urcorner
$$

First substitute $V r$ for $f$ in (16) to get

$$
\left.\left.{ }_{\llcorner} p\right\lrcorner \Gamma \Phi\left(V_{r}(p)\right)\right\urcorner
$$

This is clearly possible since $f$ and $V_{r}$ both belong to the category $\mathrm{S} / \mathrm{S}$. Then, using the logical basis so far developed, derive

$$
\left.{ }_{\llcorner} p\right\lrcorner\lceil\phi(\Phi(\operatorname{Vr}(p)) \Phi(\phi(p p)))\urcorner
$$


Now distribute the quantifiers and detach (19) from (20) to get the desired (17).

Definition. In Protothetic definitions are biconditionals or generalizations of biconditionals with the definiendum on the right. Moreover, the definiendum is either a term (when we are defining a constant) or a function (when we are defining a constant depending on several parameters). The variables in the MGQ are precisely those occurring free in the definiens and also in the definiendum. Hence definitions have the form

$$
\left.\left.\left.{ }_{\llcorner} p q \ldots\right\lrcorner\right\rangle(\psi(p q \ldots) *(p q \ldots))\right\urcorner .
$$

The requirements imposed on definitions are similar to those in other systems of logic: $\psi(p q \ldots)$ must be well formed, * is a new constant, etc. We concentrate now on the choice of parentheses in the definiendum.

The axiom of Protothetic contains only two semantical categories, the so-called basic semantic categories

$$
\text { S, S/SS. }
$$

All other semantical categories must be introduced into Protothetic by defining a constant of that category. Once a semantical category has been introduced-and only then-does the rule of procedure allow us to use variables of that category. To introduce the categories

$$
\mathrm{S} / \mathrm{S}, \mathrm{S} / \mathrm{SSS}, \mathrm{S} /(\mathrm{S} / \mathrm{S})
$$

we could use, for example, the following definitions:

$$
\begin{aligned}
& \left.\left.\left.{ }_{\llcorner} p\right\lrcorner \phi(\phi(p\llcorner u\lrcorner u\urcorner) \sim(p)\right)\right\urcorner \\
& \left.\left.{ }_{\llcorner} p q r\right\lrcorner \beta \phi(\phi(p \phi(q r)) \phi(p q r))\right\urcorner
\end{aligned}
$$

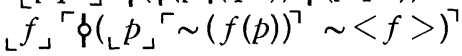

These examples serve to indicate the freedom of the rule of definition. Notice that we have used equiform parentheses in

$$
\phi(p q), \sim(p), \phi(p q r)
$$

We did this-but we would not have had to-to show that these three functors act on sentential variables. Also we chose the symbol $\phi$ in (23) since the functor defined there has several properties in common with equivalence. There is no chance to confuse the two for they have different numbers of arguments.

In (24) we chose the symbolism $\sim<f>$ to show analogy with $\sim(p)$. But here we could not have chosen the same kind of parentheses, for then we would have $f$ and $p$ in the same category. We were forced to choose a new kind of parentheses. Now if we wish to define Verum for one propositional argument we are forced to use parentheses equiform to those in the definiendum of (22):

$$
{ }_{\llcorner} p{ }_{\lrcorner} \phi(\phi(p p) \vee \mathrm{r}(p))
$$

If we took different parentheses in the definiendum-say $\neq-\neq-$ then $V r$ and $\sim$ would be in different categories; something we know is not true semantically, so we preclude it syntactically via the rule. 
At first glance one might think our analogous constants and parentheses would cause the semantical categories to coalesce. For example, if we define

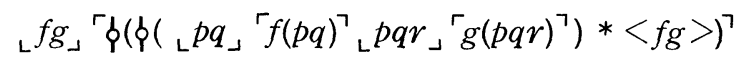

and then substitute $\phi$ from the Axiom for $f$, and $\phi$ from (23) for $g$ we would get:

$$
\left.\left.\phi\left(\phi\left({ }_{\llcorner} p q\right\lrcorner{ }_{\lrcorner} \phi(p q)^{\urcorner}{ }_{\llcorner} p q r\right\lrcorner \gamma \phi(p q r)^{\urcorner}\right) *<\phi \phi>\right)
$$

It might be supposed that $*\langle\phi \phi\rangle$ contains two occurrences of the same constant, but that is false. To see this we look at the categories of the symbols in (27). By looking at the definiens of (26) and then back to our Axiom and (23) we see $f$ is of type S/SS, $g$ of type S/SSS, and hence * is of type $\mathrm{S} /(\mathrm{S} / \mathrm{SS}, \mathrm{S} / \mathrm{SSS})$. Thus the two $\boldsymbol{\phi}^{\prime} \mathrm{s}$, although equiform, are not equisignificant.

Once a semantical category is introduced by an axiom or definition a type of parentheses is forever assigned to it. The axiom assigns the parentheses (--) to the category S/SS. The definitions (22), (23), (24), (26) dictate that a functor in the category

$\mathrm{S} / \mathrm{S}$ is always followed by parentheses of the type (-)

$\mathrm{S} / \mathrm{SSS}$ is always followed by parentheses of the type (---)

$\mathrm{S} / \mathrm{S} / \mathrm{S})$ is always followed by parentheses of the type $<->$

$\mathrm{S} /$ (S/SS, S/SSS) is always followed by parentheses of the type $<-->$.

In the future if we are defining a functor of one of these categories we must use parentheses of the indicated type. If a functor of a different type is being defined we must use different parentheses if the number of arguments are the same as one of those above.

For the purposes of substitution it does not always suffice to choose just one pair of parentheses in the definiendum. If we wanted to substitute in

$$
f(p)
$$

to get

$$
P(p q)
$$

where $\$$ represents conjunction, then a definition is necessary:

$$
\left.\left.{ }_{\llcorner} p q\right\lrcorner \beta(\rho(p q) \rho+q+(p))\right\urcorner
$$

Then we could substitute $\rho+q+$ for $f$ (they are both of type $S / S$ ) in $(28)$ to get

$$
p+q+(p)
$$

Then distribute the quantifier in (30), commute, and detach (31) to get the desired (29). This definition introduces a new category:

$$
(\mathrm{S} / \mathrm{S}) / \mathrm{S}
$$

i.e., $P$ is a functor of one sentential variable that forms a sentence forming functor of one sentential variable. Functors like $P$ in (31) are called 
many-link-functors. Many of the complications in stating the rule of definition arise from many-link-functors. We only remark here that a many-link-functor never has two sets of parentheses which are equiform, i.e., the links are enclosed in different shaped parentheses.

These informal remarks have brought out how interconnected the five parts of the rule are. Before detaching we usually distribute quantifiers; before stating a thesis of extensionality we have to give several definitions; before making a substitution we sometimes have to give a definition, and then use distribution, detachment and sometimes extensionality before obtaining the desired result. This indicates why we speak of "the rule of Protothetic" rather than "the rules of Protothetic".

The rule of Protothetic is formulated so that it automatically adjusts to the last thesis of the system. In any theory with definitions it is necessary to examine all previous definitions before adding a new one to make sure the constant has not been used before in that syntactical category.

4 The System MP. Chapters I and II dealt with general syntax, whereas the next chapter is concerned with the syntax of Protothetic. Consequently we must augment system $M$ with terms applicable specifically to Protothetic.

Since the rule of Protothetic allows us to add (under certain conditions) a new thesis $A$ immediately after thesis $B$, which is the last thesis of Protothetic, and because the rule refers to some of the theses before $B$ we need a syntactical term to express this. The term

$$
A \varepsilon \operatorname{thp}(B)
$$

which is read " $A$ is a thesis of Protothetic with respect to thesis $B$," shall be used for this purpose. The general name thp $(B)$ denotes those theses which have been derived before $B$. We use "before" here in the weak sense of "before or equal." This convention will save cumbersome phraseology later. Using this notion it is easy to define "thesis of Protothetic."

D3.4.1 $[A]: . A \varepsilon$ thp $. \equiv:\left[{ }_{\exists} B\right] . A \varepsilon \operatorname{thp}(B): A \varepsilon A$

It is important to understand that thp is a growing name. ${ }^{7}$ At the beginning the Axiom is the only thesis. The rule then allows us to add another expression (actually it is one of many possible) as a new thesis immediately after the axiom. Then thp denotes the axiom and this new thesis. At this stage the rule allows us to add a new thesis. The number of inscriptions denoted by thp is always finite, but unbounded. In effect then a thesis of Protothetic is either the axiom or something added in accordance with the rule. But the rule cannot be stated without the term thp $(B)$ and so we take it as primitive in our system MP. The axioms which this term satisfies will be stated later in this section.

As seen in the previous sections Protothetic makes heavy use of parentheses to indicate syntactical categories. Since the number of categories is unlimited we will need more than a fixed number of parentheses. Consequently we need a term to denote parentheses. One way would be to accept the general name 
prnt

as a denoter of parentheses. We shall say each of

$$
(,[,<, \neq,]
$$

is a parenthesis. We are not too concerned about the shape of parentheses and can use most any symbol as a parenthesis. For example we could write $\mathrm{p} \xi \mathrm{q}$

instead of the more customary

but then ' $p$ ' and ' $q$ ' could never be used as variables. We are more concerned with availability, for (some) new syntactical categories require new kinds of parentheses.

The term prnt does not suffice however, for we must be able to mate parentheses. Since the smallest things our syntax can refer to are words it is not capable of distinguishing left from right parentheses ${ }^{8}$ nor is it possible to say that two parentheses are symmetric. We say that the parenthesis ( is symmetric to the parenthesis ), but not to any of the parentheses

$$
<, \mathfrak{t},(, \not, t
$$

Each of the following pairs of parentheses are symmetric:

$$
(,) ;],[;<,>; f, f,
$$

so symmetry does not mean that the first is a left parenthesis and the second a right. Hence we shall take as primitive the term

$$
A \varepsilon \text { prntsym }(B)
$$

which we shall read " $A$ is a parenthesis symmetric to parenthesis $B$." Intuitively this means that $A$ is a parenthesis and $B$ is its mirror image. One easily sees that prnt can be defined in terms of prntsym:

$D 3.4 .2[A]: . A \varepsilon$ prnt . $\equiv:\left[{ }_{\exists} B\right] . A \varepsilon \operatorname{prntsym}(B): A \varepsilon A$

Hence we can just take prntsym as primitive; and we do, even though it is less intuitive.

Besides using these two primitive terms we will need to make explicit reference to the axiom of Protothetic. We give that axiom here and call it by the name $\mathfrak{A r}$ :

$$
\left.\left.\llcorner p q\lrcorner\left\lceil\left(\phi(p q)\llcorner f\lrcorner \gamma\left(f\left(p f\left(p_{\llcorner} u\right\lrcorner\ulcorner u\urcorner\right)\right)\llcorner r\lrcorner \phi(f(q r) \phi(q p))\right\urcorner\right)^{\urcorner}\right)\right\urcorner
$$

This axiom is an expression consisting of 54 words. In the next chapter we shall refer to the 1st, 4th, 5th, 9th, 10th, 11th, 12th and last of them. Moreover we need to know that the 9 th and 12 th words are symmetrical parentheses and that certain words in the axiom are not equiform. Statements $Q 7-Q 11$ use vocabulary which is not defined until the next 
chapter, but we prefer to state them here. Our assumptions about the axiom-which could either be called axioms or empirical statements-can be summarized as follows:

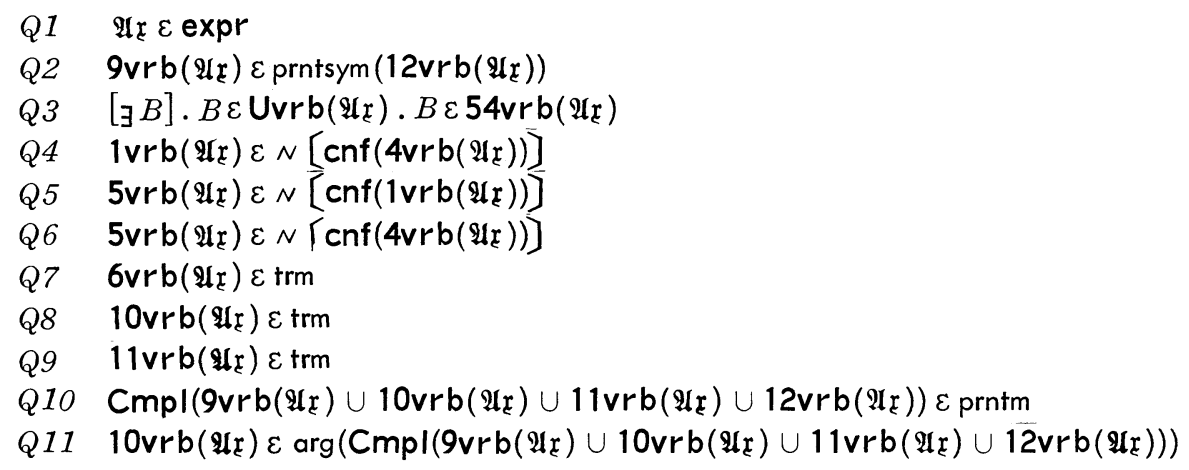

We now state the axioms which our primitive terms thp, and, prntsym

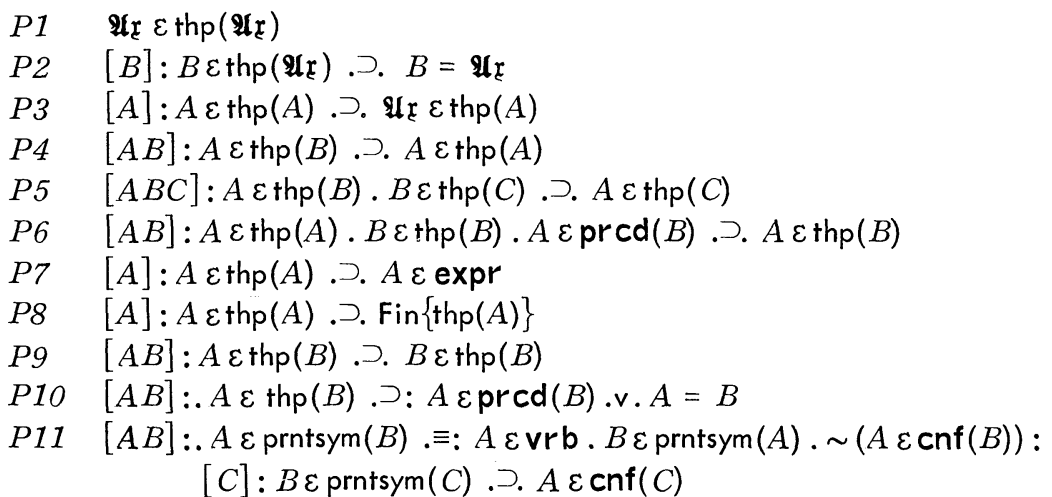

The system MP consists of system $M$ augmented by the terms

$$
\text { thp }(B) \quad \operatorname{prntsym}(B)
$$

and the axioms $P 1-P 11, Q 1-Q 11^{9}$ stated above. It is within this system that we formulate the rule of procedure of Protothetic in the next chapter.

We now make several remarks about the axioms. The denotation of thp $(B)$ cannot be axiomatically prescribed as this term has its precise meaning only after statement of the rule. The axioms stated here are all of the properties of thp $(B)$ which will be used in Chapter $V$. From $P 11$ we can derive the following theorems

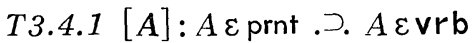

T3.4.2 $[A B]: A \varepsilon \operatorname{prntsym}(B)$.つ. $A \varepsilon$ prnt. $B \varepsilon$ prnt

$[D 3.4 .2, P 11]$

T3.4.3 $[A B]: A \varepsilon \operatorname{prntsym}(B)$.つ. $A \neq B$

T3.4.4 $[A B C]: A \varepsilon \operatorname{prntsym}(B) . B \varepsilon$ prntsym $(C)$.Ј. $A \varepsilon \operatorname{cnf}(C)$

T3.4.5 $[A B]: A \varepsilon$ prntsym $(B)$.つ. $B \varepsilon$ prntsym $(A)$

T3.4.6 $[A B]: A \varepsilon \operatorname{prntsym}(B)$.つ. $\sim(A \varepsilon \operatorname{cnf}(B))$ 
T3.4.6 is the only one of these requirements on parentheses which is really useful. It prohibits symbols like

$$
=,+, \theta, \phi
$$

from being used as parentheses. The other theses are all satisfied by any non-self-symmetric symbol, where for a symmetric parenthesis we take the mirror image. It should be remarked that if we take $P 11$ too seriously then variables and constants could only be self-symmetric symbols like

$$
\mathrm{A}, \mathrm{V}, \mathrm{T},+,=, \mathrm{U} \text {. }
$$

Since we do not wish to observe this requirement in practice, we shall use as parentheses only those symbols which one normally calls parentheses.

\section{CHAPTER IV}

To formalize a deductive theory is to stipulate directives for derivation of its theorems in purely syntactical terms. To do this it is necessary to give precise explanations (definitions) of the metalinguistic terms used in these directives.

In this chapter we give, in the most precise way that we know, a formulation of the rule of procedure of Protothetic. The rule itself can be stated quite succinctly ( $C f .6$ below), but the explanation of the terms used there will require a long sequence of Terminological Explanations (T.E.'s).

Our Terminological Explanations are nothing more than definitions of metalinguistic terms which apply expressly to Protothetic, and as such shall use, besides the terms of system $M$, only those primitive terms which are peculiar to Protothetic, viz., thp $(B)$ and prntsym $(B)$, which were explained in Chapter III, 4.

In order to make the T.E.'s more perspicuous we shall include brief (and approximate!) renderings into English and shall give some of the semantical motivation behind these definitions. Finally, we shall prove several consequences of the T.E.'s which are either interesting in themselves or useful later. This material is intended to explain and supplement the T.E.'s, but only what is in the T.E.'s is necessary for the statement of the rule.

The general methodology of the T.E.'s is to delimit the possible senseful expressions by excluding certain undesirable expressions from consideration, rather than to use inductive techniques similar to those commonly used in propositional and functional calculi to build up senseful expressions (wffs). Since we wish to construct a language as free and rich as possible, all non-essential choices are left open to the individual. Accordingly, we shall not use specific variables or constants which are prescribed ahead of time.

1 Quantifiers and Variables (TE1-12). Most theses of Protothetic are generalizations, i.e., expressions of the form

$$
\left.\left.{ }_{\llcorner} p q \ldots\right\lrcorner \psi(p q \ldots)\right\urcorner
$$


which we customarily read "for all $p, q, \ldots, \psi(p q \ldots)$." The first part of (1), viz., the part equiform to

$$
\text { ¿pq...」 }
$$

is called a (general) quantifier (more precisely, the quantifier of generalization (1)), and the remainder, viz., the part equiform to

$$
\left\ulcorner\psi(p q \ldots)^{\urcorner}\right.
$$

is called a subquantifier (more precisely, the subquantifier of generalization (1)). The words in the quantifier are called binders of the words equiform to them in the subquantifier which are called variables. Without further ado we give precise definitions of these, and some related, syntactical notions.

$$
\begin{aligned}
T E 1^{10} \quad[A]: A \varepsilon \operatorname{trm} . \equiv . \\
A \varepsilon v r b . \\
A \varepsilon N[\operatorname{prnt}] . \\
A \varepsilon N[\operatorname{cnf}(1 \operatorname{vrb}(\mathfrak{H} \mathfrak{x}))] . \\
A \varepsilon N[\operatorname{cnf}(4 \operatorname{vrb}(\mathfrak{H} \mathfrak{x}))] . \\
A \varepsilon N[\operatorname{cnf}(5 \operatorname{vrb}(\mathfrak{A} \mathfrak{x}))] . \\
A \varepsilon N[\operatorname{cnf}(\operatorname{Urb}(\mathfrak{A} \mathfrak{x}))]
\end{aligned}
$$

A term is any word which is not a parenthesis or corner, where by corner we mean any word equiform to one of the following words:

$$
\llcorner,\lrcorner,\ulcorner,\urcorner \text {. }
$$

It should be clear that corners are not parentheses, for otherwise the last four conjuncts ${ }^{11}$ of $T E 1$ would not be necessary.

TE2 $[A]: A \varepsilon$ qntf . $\equiv$.

$$
\begin{aligned}
& \operatorname{lvrb}(A) \varepsilon \operatorname{cnf}(1 \mathbf{v r b}(\mathfrak{H} \mathfrak{r})) . \\
& \text { Uvrb }(A) \varepsilon \operatorname{cnf}(4 \operatorname{vrb}(\mathfrak{H} r)) . \\
& \operatorname{Int}(A) \varepsilon \operatorname{Cmpl}(\operatorname{trm} \cap \operatorname{int}(A)) . \\
& A \varepsilon \text { non-rep }
\end{aligned}
$$

A quantifier is a string of non-equiform terms enclosed in lower cerners. Thus

$$
\llcorner p\lrcorner,\llcorner p f \psi\lrcorner,{ }_{\llcorner} q \circ f \phi_{\lrcorner}
$$

are examples of quantifiers, but the following are not:

$$
{ }_{\lrcorner} p,\llcorner\lrcorner,\left\llcornerp \left( q_{\lrcorner},\left\llcorner q q_{\lrcorner}\right. \text {. }\right.\right.
$$

By TE2.3 Int $(A)$ is an individual and hence any quantifier contains at least three words. Note that the terms in a quantifier are neither repeated or separated by commas, as there is no need to do so. Variables of different syntactical categories may be in the same quantifier, thus eliminating the need of adjacent quantifiers. Later T.E.'s will prevent constants like $\circ$ and $\phi$ from occurring in quantifiers. 
When we say quantifier we always mean general quantifier. The particular quantifier is not definable in Protothetic.

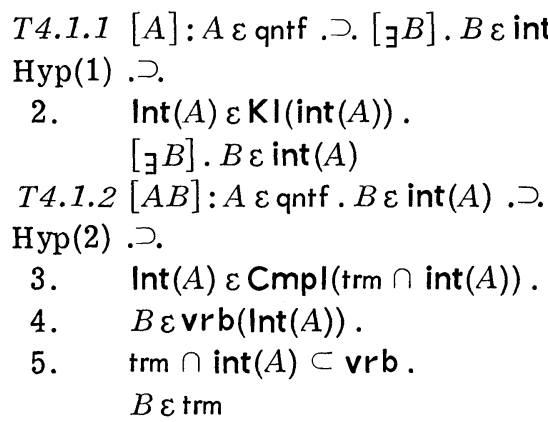

T4.1.2 $[A B]: A \varepsilon$ qntf. $B \varepsilon \operatorname{int}(A)$.Ј. $B \varepsilon \operatorname{trm}$ Hyp(2) ..

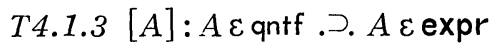

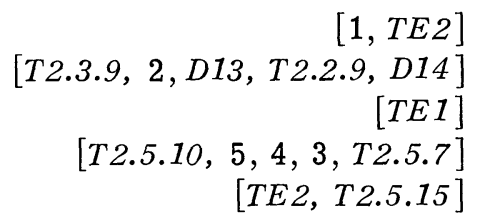

The next few lemmas are used to prove that overlapping quantifiers are identical.

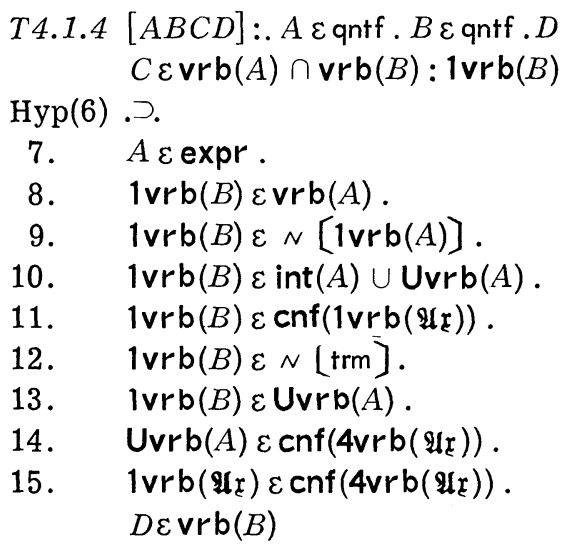

In the proof of this theorem the use of $Q 4$ is essential. It states that the words $L$ and $\lrcorner$ are not equiform.
T4.1.5 $[A B C D]: A \varepsilon q \operatorname{qnf} . B \varepsilon q \operatorname{qntf} . D \varepsilon \operatorname{pr}(C) . D \varepsilon \operatorname{vrb}(A) . C \varepsilon \operatorname{vrb}(A) \cap \operatorname{vrb}(B) . \supset$. $D \varepsilon \operatorname{vrb}(B)$

$\operatorname{Hyp}(5) . \supset$ :

6. $B \varepsilon \operatorname{expr}$.

7. $1 \mathrm{vrb}(B) \varepsilon 1 \mathrm{vrb}(B)$.

8. $\sim(C \varepsilon \operatorname{pr}(1 \operatorname{vrb}(B)))$ :

9. $\quad 1 \mathrm{vrb}(B) \varepsilon \operatorname{pr}(C) \cdot \mathrm{v} \cdot 1 \mathrm{vrb}(B)=C$ :

10. $\quad 1 \operatorname{vrb}(B) \varepsilon \operatorname{pr}(D) . v . D \varepsilon \operatorname{pr}(1 \mathrm{vrb}(B)) . \operatorname{v.} D=1 \mathrm{vrb}(B)$ :

$[T 2.2 .16,7,4, D 3, T 2.2 .9]$ $D \varepsilon \operatorname{vrb}(B)$ $[10,6, D 7,3,5, T 4.1 .4,1,2,4,5,9, D 3]$ T4.1.6 $[A B C D]: A \varepsilon$ qntf. $B \varepsilon$ qntf. $C \varepsilon \operatorname{pr}(D) . D \varepsilon \operatorname{vrb}(A)$. $C \varepsilon \operatorname{vrb}(A) \cap \operatorname{vrb}(B)$. $\supset . D \varepsilon \operatorname{vrb}(B)$ 
T4.1.8 $[A B C]: A \varepsilon$ qntf. $B \varepsilon$ qntf. $C \varepsilon \operatorname{vrb}(A) \cap \operatorname{vrb}(B) . \supset . A=B[T 4.1 .7, T 2.2 .2]$ TE3 $[A]: . A \varepsilon$ sbqntf . $\equiv$ :

$A \varepsilon$ expr-w-int :

$[C]: C \varepsilon \operatorname{hd}(A)$.Ј. $C \varepsilon \operatorname{Mtch}(\operatorname{Urrb}(\mathfrak{A r}) 5 \operatorname{vrb}(\mathfrak{A r}))$ :

$[C]: C \varepsilon \operatorname{tl}(A) . \supset . C \varepsilon \operatorname{Mtch}(5 \operatorname{vrb}(\mathfrak{A x}) \operatorname{Uvrb}(\mathfrak{A} x))$

A subquantifier is an expression enclosed by upper corners such that upper corners occurring in it are properly paired. TE3.2 stipulates that any initial segment of a subquantifier contains more left upper corners than right upper corners. Thus

$$
\ulcorner p\urcorner,\ulcorner\phi(p p)\urcorner,\ulcorner\phi(((\urcorner
$$

are subquantifiers, while

$$
\ulcorner\urcorner, r\ulcorner p\urcorner,\ulcorner p\urcorner\ulcorner q\urcorner
$$

are not. Notice that there is no requirement about parentheses being matched.

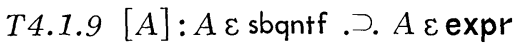

$[T E 3, T 2.5 .13]$

Now we shall prove the first (last) word of a subquantifier is a left (right) upper corner and that a subquantifier contains the same number of left and right upper corners.

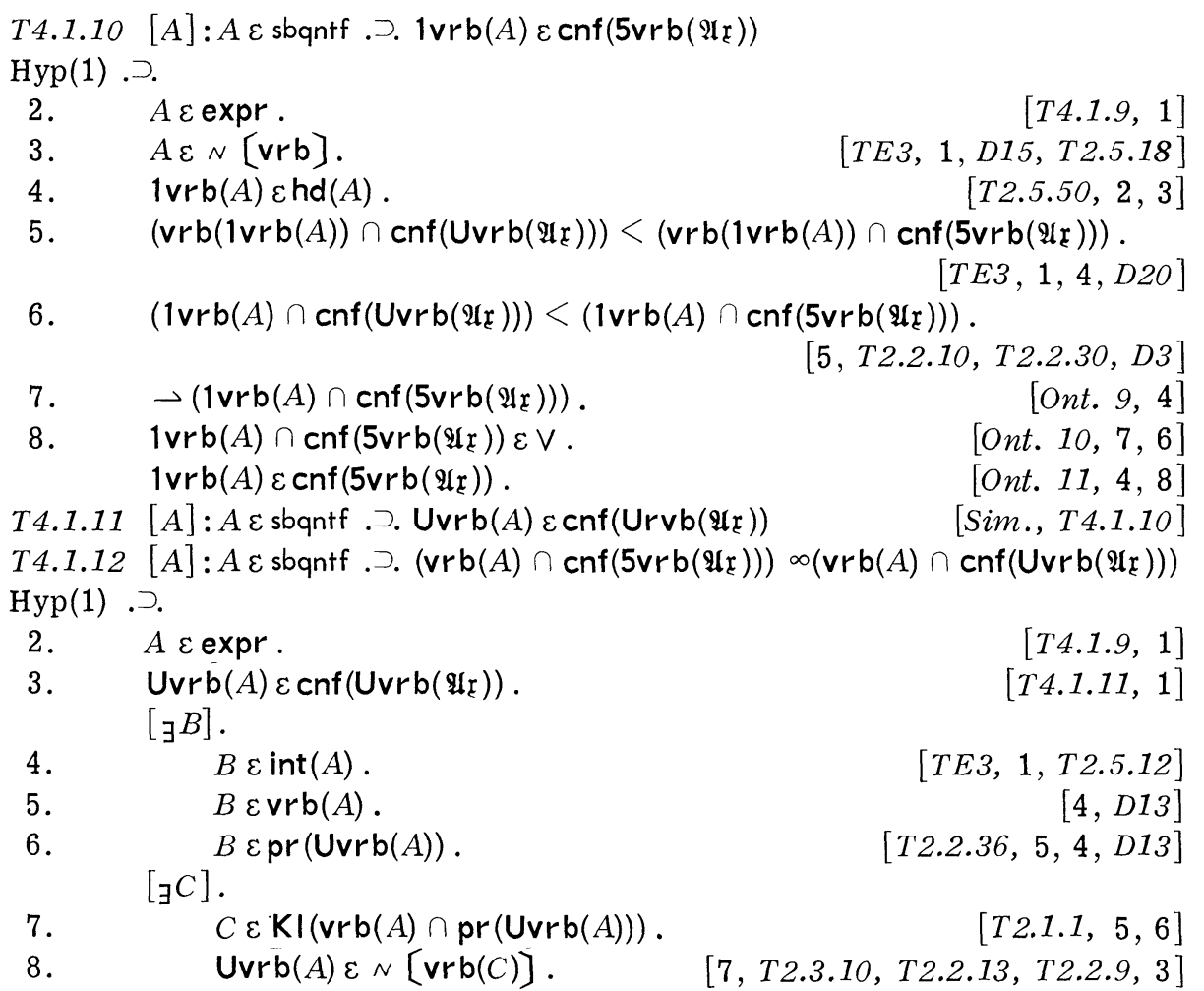


9. $\quad C \varepsilon$ expr.

$[T 2.5 .47,2,7]$

10. $\quad C \varepsilon C \operatorname{Cmpl}(\operatorname{vrb}(A) \cap \operatorname{pr}(\operatorname{Uvrb}(A)))$.

$[D 12,7,9, T 2.2 .9, T 2.4 .2, T 2.5 .2]$

11.

$C \varepsilon \operatorname{hd}(A)$.

$[T 2.5 .49,10,2,3]$

12. $\quad \operatorname{vrb}(C) \cap \operatorname{enf}(\operatorname{Uvrb}(\mathfrak{A x}))<\operatorname{vrb}(C) \cap \operatorname{enf}(5 \operatorname{vrb}(\mathfrak{A} x))$.

13. $\quad(\operatorname{vrb}(C) \cup \cup \operatorname{vrb}(A)) \circ \operatorname{vrb}(A) . \quad[7, D 1, D 5, T 2.2 .7, T 2.2 .36]$

14. $\quad \operatorname{Urb}(A) \cup(\operatorname{vrb}(C) \cap \operatorname{cnf}(\operatorname{Urb}(\mathfrak{A x})))<\operatorname{Uvrb}(\mathfrak{A r}) \cup(\operatorname{vrb}(C) \cap$ $\operatorname{cnf}(5 \mathrm{vrb}(\mathfrak{A} \mathrm{r})))$.

15. $\quad(U \operatorname{vrb}(A) \cup \operatorname{vrb}(C)) \cap(U \operatorname{vrb}(A) \cup \operatorname{enf}(U \operatorname{vrb}(\mathfrak{A r})))<$

[12, 8, Ont. 12]

$(U \operatorname{vrb}(A) \cup \operatorname{vrb}(C)) \cap(U \operatorname{vrb}(A) \cup \operatorname{cnf}(5 \operatorname{vrb}(\mathfrak{A} \mathfrak{x})))$.

16. $\quad \operatorname{vrb}(A) \cap \operatorname{cnf}(U \operatorname{vrb}(\mathfrak{A r}))<\operatorname{vrb}(A) \cap(U \operatorname{vrb}(A) \cup \operatorname{cnf}(5 \operatorname{vrb}(\mathfrak{A r})))$.

17. $\quad \operatorname{vrb}(A) \cap \operatorname{enf}(\operatorname{Uvrb}(\mathfrak{A r}))<(\operatorname{vrb}(A) \cap \operatorname{Uvrb}(A)) \cup(\operatorname{vrb}(A) \cap$ $\operatorname{cnf}(5 \mathrm{v} \overline{r b}(\mathfrak{H} \mathfrak{x})))$.

18. $\operatorname{vrb}(A) \cap \operatorname{cnf}(\operatorname{Urb}(\mathfrak{A x})) \leq \operatorname{vrb}(A) \cap \operatorname{enf}(5 \operatorname{vrb}(\mathfrak{A x}))$.

19.

$\operatorname{vrb}(A) \cap \operatorname{cnf}(5 \operatorname{vrb}(\mathfrak{A x})) \leq \operatorname{vrb}(A) \cap \operatorname{cnf}(\operatorname{Urrb}(\mathfrak{A x})) . \quad[$ Sim., 18]

$\operatorname{vrb}(A) \cap \operatorname{cnf}(5 \operatorname{vrb}(\mathfrak{A x})) \leq \operatorname{vrb}(A) \cap \operatorname{cnf}(\operatorname{Uvrb}(\mathfrak{A x}))[18,19$, Ont. 20]

The following theorems, which are not used later, are stated without proof.

T4.1.13 $[A B]: A \varepsilon$ sbqntf. $B \varepsilon$ sbqntf. $1 \mathrm{vrb}(A)=1 \mathrm{vrb}(B)$.つ. $A=B$

T4.1.14 $[A B C]: . A \varepsilon$ sbqntf. $B \varepsilon$ sbqntf. $C \varepsilon \operatorname{vrb}(A) \cap \operatorname{vrb}(B) . \supset: A \varepsilon \operatorname{ingr}(B) . v$. $B \varepsilon \operatorname{ingr}(A)$

This last theorem cannot be strengthened to identity for consider the subquantifier

$$
\ulcorner\phi(p \phi(\llcorner u\lrcorner u\urcorner p))\urcorner
$$

which contains properly a subquantifier equiform to

$$
\ulcorner u\urcorner \text {. }
$$

T4.1.14 rules out situations like

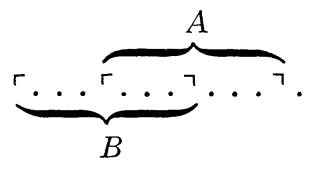

TE4 $[A B C]: A \varepsilon \operatorname{Gnrl}(B C) \equiv$.

$B \varepsilon$ qntf .

$C \varepsilon$ sbqnif.

$A \varepsilon$ Concat $(B C)$

$A$ is a generalization with quantifier $B$ and subquantifier $C$.

$T E 5 \quad[A B]: A \varepsilon \operatorname{Qntf}(B) . \equiv . A \varepsilon A .\left[{ }_{\exists} C\right] . B \varepsilon \operatorname{Gnrl}(A C)$

$A$ is the quantifier of generalization $B$. 
TE6 $[A B]: A \varepsilon \operatorname{Sbqntf}(B) . \equiv . A \varepsilon A .\left[{ }_{\exists} C\right] . B \varepsilon \operatorname{Gnrl}(C A)$

$A$ is the subquantifier of generalization $B$.

TE7 $[A]: A \varepsilon$ gnrl . $. A \varepsilon A \cdot\left[{ }_{\exists} B C\right] . A \varepsilon \operatorname{Gnrl}(B C)$

$A$ is a generalization.

A generalization is an expression of the form

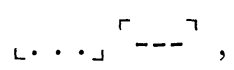

i.e., it consists of a quantifier followed by a subquantifier. We will prove that every generalization has a unique quantifier and subquantifier. However a generalization may contain many quantifiers and subquantifiers. Thus in the generalization

$$
\left.{ }_{\llcorner} p\right\lrcorner r_{\phi}\left(\phi \left(p\left\llcorner r_{\lrcorner} r^{\urcorner}\right)\left\llcorner r_{\lrcorner} r^{\urcorner}\right)^{\urcorner}\right.\right.
$$

the two expressions equiform to

$$
\llcorner\text { ᄂ }
$$

are quantifiers in the generalization, but not of the generalization. Hence "having" must be distinguished from "containing." The following are examples of expressions which are not generalizations.

$$
\left.\left.{ }_{\llcorner} p_{\lrcorner} *\ulcorner\varphi(p)\urcorner,{ }_{\llcorner} p_{\lrcorner\llcorner} q\right\lrcorner{ }^{\circ} \varphi(p q)\right\urcorner .
$$

Instead of the second of these Protothetic uses

$$
\left.{ }_{\llcorner} p q\right\lrcorner{ }{ } \varphi(p q)^{\urcorner} .
$$

Hence adjacent quantifiers will never occur in Protothetic.

$$
\begin{aligned}
& \text { T4.1.15 }[A B C D]: A \varepsilon \operatorname{Gnrl}(B C) . D \varepsilon \mathrm{Gnrl}(B C) \text {. } . A=D \quad[T E 4, D 16, T 2.5 .7] \\
& \text { T4.1.16 }[A B C]: A \varepsilon \mathrm{Gnrl}(B C) \text {.つ. } A \varepsilon \operatorname{expr} \\
& \text { T4.1.17 }[A B C]: A \varepsilon \mathrm{Gnrl}(B C) \text {. } . B \varepsilon \operatorname{ingr}(A) \\
& \text { T4.1.18 }[A B C]: A \varepsilon \mathrm{Gnrl}(B C) \text {. . } C \varepsilon \operatorname{ingr}(A) \\
& \text { T4.1.19 }[A B C]: A \varepsilon \mathrm{Gnrl}(B C) \text {.つ. } 1 \mathrm{vrb}(A)=1 \mathrm{vrb}(B) \\
& \text { T4.1.20 }[A B C]: A \varepsilon \operatorname{Gnrl}(B C) \text {.つ. Uvrb }(A)=\operatorname{Uvrb}(C) \\
& \text { T4.1.21 }[A]: A \varepsilon \mathrm{gnrl} \equiv .\left[{ }_{\exists} B\right]: B \varepsilon \operatorname{Qntf}(A) \\
& \text { T4.1.22 [A]:A } \varepsilon \text { gnrl . } \equiv .\left[{ }_{\exists} C\right] . C \varepsilon \operatorname{Sbqntf}(A) \\
& \text { T4.1.23 }[A B]: A \varepsilon \operatorname{Qntf}(B) \text {.Ј. } A \varepsilon \text { qntf } \\
& \text { T4.1.24 }[A B]: A \varepsilon \operatorname{Sbqntf}(B) \text {.つ. } A \varepsilon \text { sbqntf }
\end{aligned}
$$

After the rule of Protothetic is stated we will be able to prove partial converses of the above two theorems: If (sub)quantifier $A$ is an ingredient of a thesis of Protothetic then $A$ is the (sub)quantifier of some generalization.

$T E 8 \quad[A B]: . A \varepsilon \operatorname{Essnt}(B) . \equiv: A \varepsilon A: A \varepsilon \operatorname{Int}(\operatorname{Sbqntf}(B)) . v . A=B . A \varepsilon \operatorname{expr}$. $A \varepsilon \sim[\mathrm{gnrl}]$

$A$ is the essence of $B$.

The essence of $B$ is the interior of the subquantifier of $B$ if $B$ is a generalization, and $B$ otherwise. 
T4.1.25 $[A]: A$ \& gnrl .つ. $\operatorname{vrb}(\operatorname{Essnt}(A)) \circ \operatorname{int}(\operatorname{Sbqntf}(A))$

$\operatorname{Hyp}(1) . \supset$ :

$\begin{array}{llr}\text { 2. } & {[B]: B \varepsilon E \operatorname{Esnt}(A) . \equiv . B \varepsilon \operatorname{lnt}(\operatorname{Sbqntf}(A)):} & {[1, T E 8]} \\ 3 . & \operatorname{Essnt}(A) \circ \operatorname{Int}(\operatorname{Sbqntf}(A)) . & {[2]} \\ \text { 4. } & \operatorname{vrb}(\operatorname{Essnt}(A)) \circ \operatorname{vrb}(\operatorname{Int}(\operatorname{Sbqntf}(A))) . & {[3]} \\ & \operatorname{vrb}(\operatorname{Essnt}(A)) \circ \operatorname{int}(\operatorname{Sbqntf}(A)) \quad[4, T 2.5 .22, T 2.3 .9, D 13, T 2.2 .9, D 14]\end{array}$ TE9 $[A B]: A \varepsilon \operatorname{bd}(B) . \equiv . A \varepsilon \operatorname{int}(\operatorname{Qntf}(B))$

$A$ is a binder in $B$.

A binder is a word in the quantifier of a generalization. This T.E. is purely abbreviatory yet frequently used.

T4.1.26 $[A B]: A \varepsilon b d(B)$. . $A \varepsilon \operatorname{vrb}(B)$

$\operatorname{Hyp}(1) . \supset$.

2. $A \varepsilon \operatorname{int}\left(Q_{\operatorname{ntf}}(B)\right)$.

$[T E 9,1]$

$\left[{ }_{3} C\right]$.

3. $\quad C \varepsilon \operatorname{Qntf}(B)$.

$[2, D 13]$

4. $A \varepsilon \operatorname{vrb}(C)$.

$[2,3, D 13]$

$\left[{ }_{\exists} D\right]$.

5. $\quad B \varepsilon \mathrm{Gnrl}(C D)$.

$[3, T E 5]$

6. $C \varepsilon$ ingr $(B)$.

$A \varepsilon \operatorname{vrb}(B)$

T4.1.27 $[A B]: A \varepsilon b d(B)$. $\supset .\left[{ }_{\exists} C\right] . C \varepsilon$ qntf . $A \varepsilon \operatorname{int}(C)$

5. $T E 4, T 2.5 .30]$

$[T 2.4 .10,4,6]$ $\operatorname{Hyp}(1)$..

2. $A \varepsilon \operatorname{int}\left(Q_{n t f}(B)\right)$.

$[T E 9,1]$ $\left[{ }_{\exists} C\right]$.

3. $A \varepsilon \operatorname{int}(C)$.

$[2, D 13]$

4. $C \varepsilon \operatorname{Qntf}(B)$.

5. $C \varepsilon$ qntf.

$[4, T 4.1 .23]$ $\left[{ }_{\exists} C\right] . A \varepsilon \operatorname{int}(C) . C \varepsilon$ qntf

T4.1.28 $[A B]: A \varepsilon$ bd $(B)$.つ. $B \varepsilon$ gnrl

It is important to remember that binders are not just words in quantifiers, but words in quantifiers of generalizations.

T4.1.29 $[A B C]: A \varepsilon \operatorname{bd}(B) . C \varepsilon \operatorname{bd}(B) . A \varepsilon \operatorname{cnf}(C)$. $. A=C$ $\operatorname{Hyp}(3)$.. .

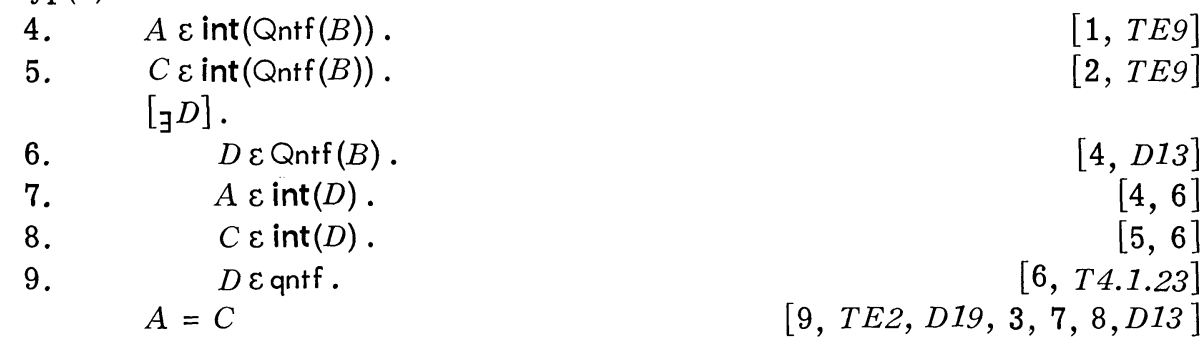

$T E 10 \quad[A B C]: . A \varepsilon \operatorname{var}(B C) . \equiv$ :

$B \varepsilon$ bd $(C)$.

$A \varepsilon \operatorname{int}(\operatorname{Sbqntf}(C))$.

$A \varepsilon \operatorname{cnf}(B)$ :

$[D E]: D \varepsilon \operatorname{ingr}(C) . E \varepsilon \operatorname{bd}(D) . A \varepsilon \operatorname{cnf}(E) . A \varepsilon \operatorname{vrb}(D) . \supset . D=C$ 
$A$ is a variable bound by $B$ in generalization $C$. This very important notion will be used constantly, so we give several examples. Consider the following generalization

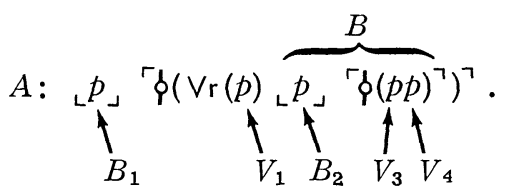

In this example we have pointed to certain words to name them. This will avoid cumbersome description like " $B_{1}$ is the name of the second word of $A$," " $B$ is the name of the Complex of the 11th through 20th words of $A$." Referring now to this diagram we can say $V_{1} \varepsilon \operatorname{var}\left(B_{1} A\right)$, and moreover $V_{1}$ is the only variable of $A . V_{3} \varepsilon \operatorname{var}\left(B_{2} B\right)$ and $V_{4} \varepsilon \operatorname{var}\left(B_{2} B\right)$, so it should be clear that one binder can bind several variables. But neither $V_{3}$ nor $V_{4}$ is a variable in $A$. Since quantifiers contain no equiform words no variable can be bound by more than one binder. This prompts us to prove

T4.1.30 $[A B C D E]: A \varepsilon \operatorname{var}(D C) . B \varepsilon \operatorname{var}(E C) . A \varepsilon \operatorname{cnf}(B)$. $. D=E$ $\operatorname{Hyp}(3)$.. .

$$
\begin{array}{ll}
\text { 4. } & D \varepsilon b d(C) . \\
\text { 5. } & A \varepsilon \operatorname{cnf}(D) . \\
\text { 6. } & E \varepsilon \operatorname{bd}(C) . \\
\text { 7. } & B \varepsilon \operatorname{cnf}(E) . \\
\text { 8. } & D \varepsilon \operatorname{cnf}(E) . \\
& D=E
\end{array}
$$

T4.1.32 $[A B C]: A \varepsilon \operatorname{var}(B C)$.つ. $C \varepsilon \mathrm{gnrl}$

$T E 11[A B C]: . A \varepsilon \operatorname{cnvar}(B C) . \equiv: A \varepsilon A .\left[{ }_{\exists} D\right] . A \varepsilon \operatorname{var}(D C) . B \varepsilon \operatorname{var}(D C)$

$A$ is an equiform variable with $B$ in $C$.

$T E 12 \quad[A B]: A \varepsilon \operatorname{var}(B) . \equiv . A \varepsilon \operatorname{cnvar}(A B)$

$A$ is a variable in the generalization $B$. As an example consider the generalization $A$ :

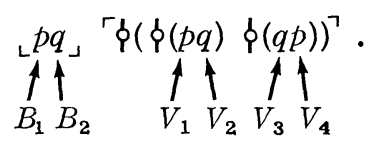

We have

$$
\begin{aligned}
& V_{1} \varepsilon \operatorname{cnvar}\left(V_{4} A\right), \quad V_{2} \varepsilon \operatorname{cnvar}\left(V_{3} A\right) \\
& V_{1} \varepsilon \operatorname{var}\left(B_{1} A\right), \quad V_{3} \varepsilon \operatorname{var}\left(B_{2} A\right) \\
& V_{i} \varepsilon \operatorname{var}(A), \quad(i=1,2,3,4)
\end{aligned}
$$

The following theorems are obvious:

T4.1.33 $[A B C]: A \varepsilon$ cnvar $(B C)$.つ. $A \varepsilon$ cnvar $(A C)$

T4.1.34 $[A B C]: A \varepsilon \operatorname{cnvar}(B C)$.つ. $A \varepsilon \operatorname{var}(C)$

T4.1.35 $[A B]: A \varepsilon \operatorname{var}(B)$. D. $B \varepsilon \mathrm{gnrl}$ 
T4.1.36 $[A B]: A \varepsilon \operatorname{var}(B) . \equiv .\left[{ }_{\exists} C\right] . A \varepsilon \operatorname{var}(C B)$

[TE12, TE 11]

T4.1.37 $[A B C]: A \varepsilon$ cnvar $(B C)$..$B \varepsilon$ cnvar $(A C)$

T4.1.38 $[A B C]: A \varepsilon \operatorname{cnvar}(B C)$. . $A \varepsilon \operatorname{enf}(B) \quad[T E 11, T E 10, T 2.2 .20, T 2.2 .21]$

T4.1.39 $[A B]: A \varepsilon \operatorname{var}(B)$.ว. $B \varepsilon \mathrm{trm} \quad[T 4.1 .36, T E 10, T E 9, T 4.1 .2, T E 1]$

2 Functions and Arguments (TE13-29). We previously remarked that almost all theses of Protothetic are generalizations of the form

$$
\left.{ }_{\llcorner} p q \ldots\right\lrcorner\ulcorner\psi(p q \ldots)\urcorner .
$$

More precisely, the essence of (1) is usually a many-link-function, i.e., an expression of the form

$$
\Phi \neq p q \ldots \neq t f g \ldots+\ldots(f p \ldots)
$$

In (2) the links, i.e., the expressions like

$$
\neq p q \ldots \neq,+f g \ldots+,(f p \ldots)
$$

are called parenthemes and consist of an expression enclosed between symmetric parentheses. Each parentheme can be uniquely decomposed into an expression of the form

$$
\left\langle a_{1} a_{2} \ldots a_{\mathrm{n}}\right\rangle
$$

where each $a_{i}$ is a term, generalization, or another function like (2). These $a$ 's are called the arguments of the parentheme (4). Besides these notions we shall develop vocabulary for comparing parenthemes and the arguments of different parenthemes.

$T E 13$

$$
\begin{aligned}
& {[A]: A \varepsilon \text { prntm } . \equiv:} \\
& A \varepsilon \operatorname{expr}-\mathrm{w}-\mathrm{int}: \\
& {[C]: C \varepsilon \mathrm{gd}(A) . \supset . C \varepsilon \operatorname{Mtch}(\operatorname{Uvrb}(A) \operatorname{1vrb}(A)):} \\
& {[C]: C \varepsilon \operatorname{tl}(A) . \supset . C \varepsilon \operatorname{Mtch}(1 \mathrm{vrb}(A) \operatorname{Uvrb}(A)):} \\
& \text { 1vrb }(A) \varepsilon \operatorname{prntsym}(\operatorname{Uvrb}(A))
\end{aligned}
$$

$A$ is a parentheme. In a parentheme the first and last word are symmetric parentheses, and those parentheses within $A$, which are equiform to either the first or last parentheses of $A$, are properly mated. As examples of parenthemes consider

$$
\left.(p), \neq \varphi^{*} \neq,<_{\llcorner} u\right\lrcorner\left\ulcorner f(u)^{\urcorner}\right)(>.
$$

One should also see the remarks concerning subquantifiers (TE3) as the T.E.'s are quite similar. The following theorems are analogous to those following $T E 3$ and so no proofs are given.

T4.2.1 $[A]: A \varepsilon$ prntm .つ. $1 \mathrm{vrb}(A) \varepsilon$ prnt

T4.2.2 $[A B]: A \varepsilon$ prntm. $B \varepsilon$ prntm . 1vrb $(A) \varepsilon \operatorname{vrb}(B)$.つ. $A \varepsilon$ ingr $(B)$

T4.2.3 $[A B]: A \varepsilon$ prntm. $B \varepsilon \operatorname{prntm} .1 \mathrm{vrb}(A)=1 \mathrm{vrb}(B)$.つ. $A=B$

T4.2.4 $[A B]: A \varepsilon$ prntm .Ј. $A \varepsilon$ expr

We cannot prove yet that if two parenthemes have a word in common then one is an ingredient of the other, for consider 


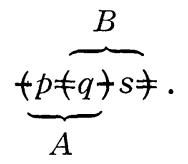

Here parenthemes $A$ and $B$ are not disjoint, yet neither is part of the other. TE14 [Aa]:A $\varepsilon \operatorname{Fnct}(a) . \equiv: 1 \mathrm{vrb}(A) \varepsilon \operatorname{trm}:$

$$
\begin{aligned}
& {[C]: C \& a . \supset . C \text { \& prntm : }} \\
& A \varepsilon C \mathrm{Cmpl}(1 \mathrm{vrb}(A) \cup a)
\end{aligned}
$$

$A$ is a function with parenthemes $a$.

$$
T E 15 \quad[A]: A \varepsilon \text { fnct . } \equiv . A \varepsilon A \cdot\left[{ }_{\exists} a\right] . A \varepsilon \operatorname{Fnct}(a) . !\{a\}
$$

$A$ is a function. A function is of the form

$$
\Phi \neq \ldots \neq t \ldots+\ldots . . . .) \text {. }
$$

The conjunct $!\{a\}$ in TE15 prevents terms from being functions. Hence we know a function has at least one parentheme. The many-link-functions we spoke of earlier are functions with more than one parentheme.

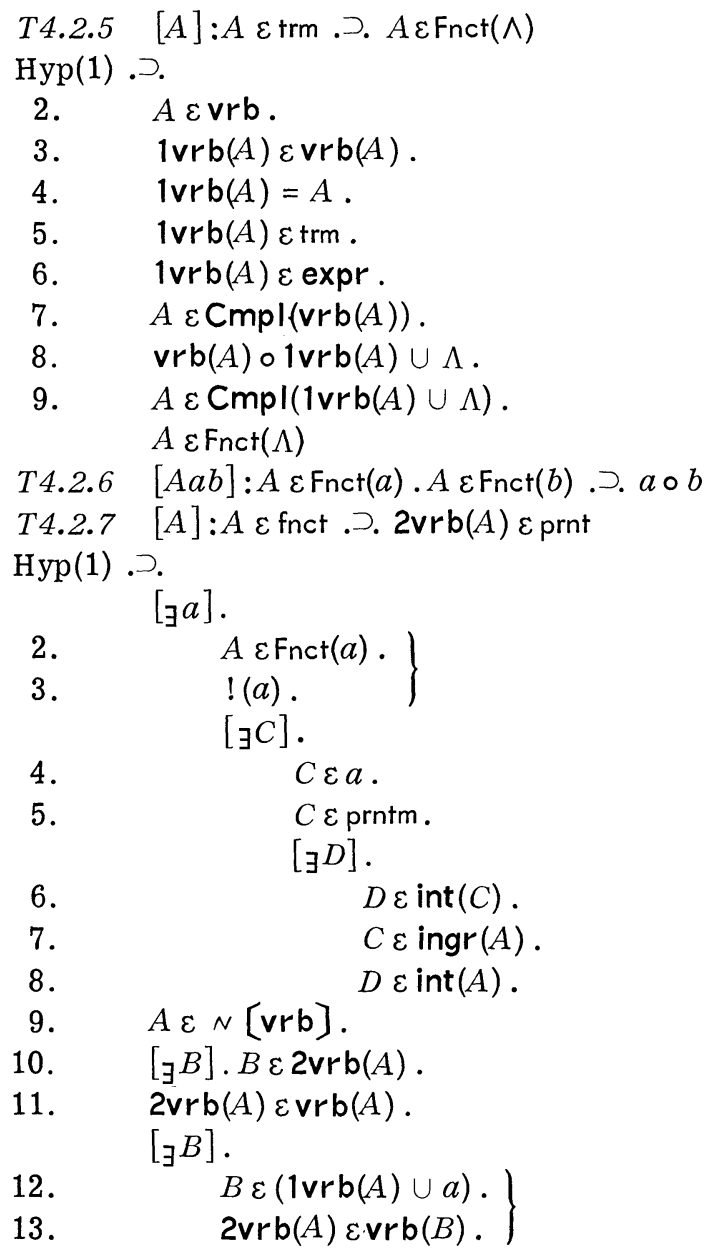

T4.2.7 $[A]: A \varepsilon$ fnct .つ. $2 \mathrm{vrb}(A) \varepsilon \mathrm{prnt}$

$\operatorname{Hyp}(1)$..
2.
3 .
$!(a)$
$C \varepsilon a$.
$C \varepsilon$ prntm.
$\left.\begin{array}{ll}\text { 12. } & B \varepsilon(1 \mathrm{vrb}(A) \cup a) . \\ \text { 13. } & 2 \operatorname{vrb}(A) \varepsilon \operatorname{vrb}(B) .\end{array}\right\}$

$[T E 1,1]$

$[1, T 2.2 .27, T 2.2 .30, D 3]$ $[3,2, T 2.2 .10]$

$[3, T 2.4 .2, T 2.2 .9]$

$[T 2.5 .8,6,4]$ $[4,3, T 2.2 .10]$ $[7,8]$ $[T E 14,5,9]$ [TE14] 
$\begin{array}{ll}\text { 14. } & B \varepsilon a . \\ \text { 15. } & \operatorname{lvrb}(A) \varepsilon N[\operatorname{vrb}(B)] .\end{array}$

$[12,13, T 2.2 .39]$

16. $B \varepsilon$ prntm.

$[T E 14,14,2]$

17. $B \varepsilon \operatorname{ingr}(A)$.

18. $2 \operatorname{vrb}(A)=1 \mathrm{vrb}(B)$.

19. $\quad 1 \mathrm{vrb}(B) \varepsilon$ prnt.

$[14,2, T E 14, T 2.5 .9]$
$[13,15,17, T 2.2 .41]$

$2 \mathrm{vrb}(A) \varepsilon \mathrm{prnt}$

$[16, T 4.2 .1]$

$[18,19]$

T4.2.8 $[A]: A \varepsilon$ fnct .つ. Uvrb $(A) \varepsilon$ prnt

[Sim., T4.2.7]

$T E 16 \quad[A B]: A \varepsilon \operatorname{prntm}(B) . \equiv . A \varepsilon A .\left[{ }_{\exists} a\right] . B \varepsilon \operatorname{Fnct}(a) . A \varepsilon a$

$A$ is a parentheme of the function $B$.

T4.2.9 $[A B]: A \varepsilon \operatorname{prntm}(B)$.つ. $B \varepsilon$ fnct

[TE16,TE15]

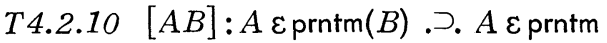

[TE 16, TE14]

Clearly a function can have several parenthemes, but it is not so clear that a parentheme can be a parentheme of several functions. Consider the function $A$ :

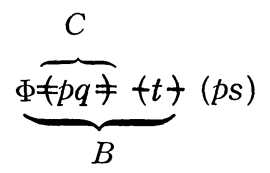

$C$ is a parentheme of $A$ and also of the function $B$.

TE17 $[A a]: . A \varepsilon P-\arg (a) . \equiv:$

$A \varepsilon$ prntm :

$[C]: C \varepsilon a . \supset . C \varepsilon$ trm $\cup$ gnrl $\cup$ fnct :

$\operatorname{lnt}(A) \varepsilon \operatorname{Cmpl}(a)$

$A$ is a parentheme with arguments $a$.

$T E 18 \quad[A B]: A \varepsilon \arg (B) . \equiv . A \varepsilon A .\left[{ }_{\exists} a\right] . B \varepsilon \mathrm{P}-\arg (a) . A \varepsilon a$

$A$ is an argument of parentheme $B$. The most important thing to remember is that arguments can only be terms, generalizations or functions.

T4.2.11 $[A B]: A \varepsilon \arg (B)$.つ. $A \varepsilon$ trm $\cup$ gnrl $\cup$ fnct

[TE 18, TE17]

$T 4.2 .12[A B]: A \varepsilon \arg (B)$.Ј. $B \varepsilon$ prntm

[TE 18, TE17]

Only parenthemes have arguments. We will not speak of functions having arguments, but only of their parenthemes having arguments. We cannot prove that all parenthemes have arguments, for consider the parentheme:

$$
\text { (r). }
$$

$r$ is the only thing which has a chance of being an argument, but it is neither a term, generalization nor function. So this parentheme has no arguments.

The requirement that arguments be terms, generalizations or functions is quite natural from a semantical point of view; we certainly do not want 
corners, parentheses, quantifiers or any meaningless expressions to occur as arguments. Syntactically it is easy to say to which of these three classes an argument belongs. Terms are always single letters, but not corners or parentheses. Generalizations always begin with $L$ and end with 7. Because of the condition on matching corners we can always find the end of a generalization. Functions always consist of a term followed by a number of parenthemes. All of this is intended to convince one that we do not need commas between the arguments. Consider, for example, the following parentheme $A$ :

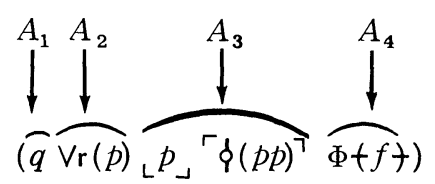

The first argument must begin with the first word in $\operatorname{lnt}(A)$, i.e., $q$. Since the third word of $A$ is a term, the first argument of $A$ must be just the second word of $A$. As the 4 th word of $A$ is a parenthesis the $3 \mathrm{rd}$ word cannot be an argument by itself. So the 4th word is part of the second argument and moreover it begins a parentheme of the function which must be the second argument of $A$. This parentheme ends with the 6th word of $A$. As the 7th word is equiform to $L$ it cannot begin a new parentheme of the function which is the second argument and so begins the third argument. Since words equiform to $L$ can only start generalizations the third argument can only be a generalization. Going to the end of $A_{3}$ we find that the next word is a term and so is either an argument by itself or begins a function. Since the word after $\Phi$ is a parenthesis we see that the last argument is $A_{4}$. This argument should convince one of the plausibility of the following theorem which states that parenthemes with arguments have those arguments uniquely.

T4.2.13 $[A a b]: A \varepsilon p-\arg (a): A \varepsilon p-\arg (b)$.つ. $a \circ b$

We also have

T4.2.14 $[A B C]: A \varepsilon \arg (B) . A \varepsilon \arg (C)$.Ј. $B=C$

We now begin a sequence of T.E.'s which will enable us to discuss corresponding arguments of parenthemes and analogous functions. The semantical reason for these syntactic requirements is as follows: We want the number of arguments and shape of the parentheses of a parentheme to indicate the syntactical categories of its arguments.

The idea is to say-and this is the purpose of the next paragraph-that corresponding arguments enclosed in the same type of parentheses are of the same syntactical category. Thus for example when we write

$$
\psi(p q), \psi\left(\varphi_{\llcorner} u_{\lrcorner}\ulcorner u\urcorner\right)
$$

we want $p$ and $\varphi$, and also $q$ and $\llcorner u\lrcorner\ulcorner u\urcorner$ to belong to the same category.

We avoid things like

$$
f(p), \Phi(f)
$$


Since we could conclude that $p$ and $f$ and also $f$ and $\Phi$ are in the same category. Hence $f$ and $p$ are in the same category. But a functor and an argument of its parentheme being in the same category smacks of the Russell Antinomy.

$T E 19 \quad[A B]: A \varepsilon \operatorname{simprntm}(B) . \equiv$.

$A \varepsilon$ prntm.

$B \varepsilon$ prntm.

$1 \mathrm{vrb}(A) \varepsilon \operatorname{cnf}(1 \mathrm{vrb}(B))$.

$\arg (A) \infty \arg (B)$

$A$ is a parentheme similar to $B$. Because of T4.2.12, i.e., the only things with arguments are parenthemes, it seems TE 19.2 could be dropped. This is indeed the case if we knew parentheme $A$ had any arguments. Consider $\langle L\rangle$ and $\langle\langle L\rangle$. The first of these is a parentheme with no arguments. The second also has no arguments, since it is not even a parentheme. This establishes the independence of TE19.2 (or TE 19.1).

The following parenthemes are similar,

$$
\left.(p q),\left({ }_{\llcorner} p\right\lrcorner\lceil(p p)\urcorner s\right),(f(t) \Phi<f>)
$$

but none of these is similar to any of the following

$$
(p),(p q r),+\llcorner p)
$$

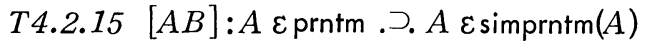

T4.2.16 $[A B]: A \varepsilon \operatorname{simprntm}(B)$.つ. $B \varepsilon \operatorname{simprntm}(A)$

[TE19, Ont. 14, T2.2.18]

[TE19, Ont. 15, T2.2.20]

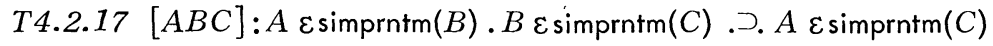

TE $20 \quad[A B C D]: A \varepsilon \operatorname{Anarg}(B C D) . \equiv$.

[TE19, Ont. 16, T2.2.21]

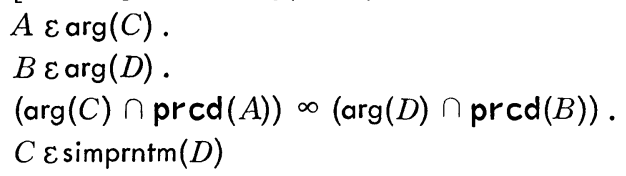

$A$ is an analogous argument to $B$, which are arguments of $C$ and $D$ respectively. Intuitively two arguments are analogous when they are both the n-th argument of their corresponding parenthemes. For example in the similar parenthemes

$$
\left(p f(q) u_{\llcorner}\left\ulcorner u^{\urcorner}\right)\right.
$$

and

$$
(t * \Phi<f>)
$$

$p$ is analogous to $t, f(q)$ to $*$ and $\llcorner u\lrcorner\ulcorner u\urcorner$ to $\Phi<f\rangle$.

T4.2.18 $[A B C D] . A \varepsilon \operatorname{Anarg}(B C D)$..$C \varepsilon$ prntm. $D \varepsilon$ prntm

$[T E 20, T E 19]$

T4.2.19 $[A B C D]: A \varepsilon \operatorname{Anarg}(B C D)$. ว. $A \varepsilon A \operatorname{Anarg}(A C C)$

[TE20, Ont. 14, T4.2.18, T4.2.15]

T4.2.20 $[A C]: A \varepsilon \arg (C)$. . A $A$ Anarg $(A C C)[T E 20$, Ont. 14, T4.2.15, T4.2.12] 
TE21 $[A B C D E F]: A \varepsilon$ Anfnct $(B C D E F) . \equiv$.

$C \varepsilon$ Concat $(A E) . A \varepsilon \bar{A}$

$D \varepsilon$ Concat $(B F)$.

$E \varepsilon \operatorname{simprntm}(F)$.

$C \varepsilon$ fnct .

$D \varepsilon$ fnct

$T E 22[A B C D]: A \varepsilon \operatorname{Anfnct}(B C D) . \equiv . A \varepsilon A .\left[{ }_{3} E F\right] . A \varepsilon \operatorname{Anfnct}(B C D E F)$

$A$ is an analogous functor to $B$ in $C$ and $D$ respectively. By a functor we mean either a function or term which is followed by a parentheme. Two functors are analogous when the parenthemes following them are similar. For example, in the functions

$$
\left.\operatorname{Vr}(p q), \Phi+f+\left(t_{\llcorner} u\right\lrcorner\ulcorner u\urcorner\right)
$$

the functors $V r$ and $\Phi f f t$ are analogous, but are not analogous to any functor of the functions

$$
f(p), \operatorname{Vr}[f q], \chi+p q+(s t u) .
$$

T4.2.21 $[A B C D]: A \varepsilon$ Anfnct $(B C D)$..$A \varepsilon$ Anfnct (ACC)

[TE22, TE21]

$T E 23 \quad[A B C D]: . A \varepsilon \operatorname{An}(B C D) . \equiv: A \varepsilon A: A \varepsilon \operatorname{Anarg}(B C D)$. . $A \varepsilon \operatorname{Anfnct}(B C D)$

$A$ is analogous to $B$ which are in $C$ and $D$ respectively iff $A$ and $B$ are either analogous arguments of parenthemes $C$ and $D$ respectively or analogous functors of $C$ and $D$ respectively.

T4.2.22 $[A B C D]: A \varepsilon \operatorname{An}(B C D)$. . $A \varepsilon \operatorname{An}(A C C) \quad[T E 23, T 4.2 .19, T 4.2 .21]$

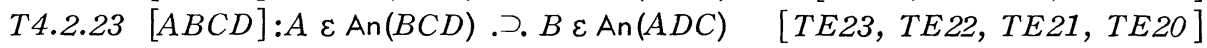

$T E 24 \quad[A B]: A \varepsilon \operatorname{larg}(B) . \equiv A \varepsilon \operatorname{Anarg}(10 \mathrm{vrb}(\mathfrak{H} \mathfrak{x}) B \mathrm{Cmpl}(9 \mathrm{vrb}(\mathfrak{H} \mathfrak{x}) \cup$ $10 \mathrm{vrb}(\mathfrak{A r}) \cup 11 \mathrm{vrb}(\mathfrak{H r}) \cup 12 \mathrm{vrb}(\mathfrak{A r})))$

$A$ is the first argument of parentheme $B$.

TE25 $[A B]: A \varepsilon 2 \arg (B) . \equiv A \varepsilon \operatorname{Anarg}(11 \mathrm{vrb}(\mathfrak{A r}) B \mathrm{Cmpl}(9 \mathrm{vrb}(\mathfrak{H} \mathfrak{z}) \cup$ $10 \mathrm{vrb}(\mathfrak{A x}) \cup 11 \mathrm{vrb}(\mathfrak{A x}) \cup \overline{12 v r b}(\mathfrak{A x})))$

$A$ is the second argument of parentheme $B$. It is important to remember that we are speaking of the arguments of very special parenthemes, viz., those similar to

$(p q)$

which is equiform to the Complex of the 9th through 12th words of our axiom $\mathfrak{A}$.

TE26 $[A B]: . A \varepsilon \operatorname{lequl}(B) . \equiv: 1 \mathrm{vrb}(B) \varepsilon \operatorname{enf}(6 \mathrm{vrb}(\mathfrak{A r})):$

$\left[{ }_{\exists} C\right] . A \varepsilon \operatorname{larg}(C) . B \varepsilon$ Concat $(1 \mathrm{vrb}(B) C): A \varepsilon A$

$A$ is the first equivalence of $B$.

TE27 $[A B]: . A \varepsilon 2 \operatorname{eqvl}(B) . \equiv: 1 \operatorname{vrb}(B) \varepsilon \operatorname{cnf}(6 \operatorname{vrb}(\mathfrak{A r}))$ :

$\left[{ }_{\exists} C\right] . A \varepsilon 2 \arg (C) . B \varepsilon$ Concat $(1 \mathrm{vrb}(B) C): A \varepsilon A$

$A$ is the second equivalence of $B . B$ is a function of the form 


$$
\phi(--),
$$

where $\phi$ is to be interpreted as the biconditional.

T4.2.24 $[A B]: A \varepsilon$ lequl $(B)$..$B \varepsilon$ fnct

$\operatorname{Hyp}(1) . \supset$.

2. $\quad 1 \mathrm{vrb}(B) \varepsilon \operatorname{enf}(6 \mathrm{vrb}(\mathfrak{A r}))$.

$[1, T E 26]$

3. $1 \mathrm{vrb}(B) \varepsilon \mathrm{trm}$.

$[2, T E 1, Q 7]$

$\left[{ }_{\exists} C\right]$.

$\left.\begin{array}{ll}\text { 4. } & A \varepsilon \operatorname{larg}(C) . \\ \text { 5. } & B \varepsilon \text { Concat }(1 \mathrm{vrb}(B) C) .\end{array}\right\}$

$[1, T E 26]$

6. $\quad C \varepsilon$ prntm.

7. $\quad B \varepsilon \operatorname{Fnct}(C)$.

[4, TE24, TE20, TE19]

$B \varepsilon$ fnct

$[T E 14,3,6,5]$

$[6,7, T E 15]$

TE28 $[A B]: A \varepsilon \operatorname{ltrm}(B) . \equiv . A \varepsilon$ lequl $($ Essnt $(B))$

$A$ is the first term of $B$.

TE29 $[A B]: A \varepsilon 2 \operatorname{trm}(B) . \equiv . A \varepsilon 2 \operatorname{equl}(\operatorname{Essnt}(B))$

$A$ is the second term of $B$. There are two cases to consider here. If $B$ is a generalization then it is of the form

$$
\text { L... }\ulcorner\oint(--)\urcorner
$$

where the first dash indicates its first term and the second dash its second term. If $B$ is not a generalization then $\operatorname{Essnt}(B)=B$ and hence is of the form

$$
\phi(--) \text {. }
$$

These forms are extremely important in Protothetic. All definitions have one of these two forms, although the second is used only in defining constants. All theses of extensionality have the first form.

3 Syntactical Categories (TE30-33). No one today would consider the expression

\section{$f(f)$}

as meaningful, yet there is still considerable debate as to how to preclude such expressions from arising in formal languages. Leśniewski's solution was to introduce the concept of semantical category into his logical theories. The main semantical criteria for two expressions to be of the same semantical category is that they be intersubstitutable in all contexts. We cannot use this as a criteria for defining semantical categories, but it is the main motivational force behind what we now do.

TEзо $[A B]: A \varepsilon \operatorname{ingrthp}(B) . \equiv . A \varepsilon A .\left[{ }_{3} C\right] . C \varepsilon \operatorname{thp}(B) . A \varepsilon \operatorname{ingr}(C)$

$A$ is an ingredient of a thesis of Protothetic with respect to $B$. This term is purely abbreviatory so we make no further comment. 
TE31 $[A B]: . A \varepsilon \operatorname{frp}(B) . \equiv: A \varepsilon A: A \varepsilon$ thp $(B) . v \cdot\left[{ }_{\exists} C\right] . C \varepsilon$ ingrthp $(B) . C \varepsilon$ sbqntf . $A \varepsilon \operatorname{lnt}(C) \cdot v \cdot\left[{ }_{\exists} C\right] . C \varepsilon$ ingrthp $(B) . A \varepsilon \operatorname{larg}(C) \cdot v \cdot\left[{ }_{\exists} C\right] . C \varepsilon$ ingrthp $(B)$. $A \varepsilon 2 \arg (C)$

$A$ is a (propositional) phrase with respect to $B$. This is a start toward building up the syntactical category of propositions. Clearly we should include all theses and essences of generalizations. We also include the arguments of parenthemes similar to

i.e., both $p$ and $q$ are to be called propositions. One should realize that $\operatorname{frp}(B)$ does not include everything which is in the syntactical category of propositions, for consider the definition of Verum:

$$
\left.\left.{ }_{\llcorner} p\right\lrcorner{ } \phi(\phi(p p) \vee r(p))\right\urcorner
$$

It is not the case that the $p$ in $\operatorname{Vr}(p)$ is a phrase. Since we want it to be of the same category as the $p$ 's in $\phi(p p)$ we must enlarge our notions so that equiform variables are of the same category.

Immediately after the above definition we could make the following definition.

$$
\left.\left.{ }_{\llcorner} p\right\lrcorner \Gamma_{\phi}\left(\llcorner f\lrcorner r_{f(p)}\right)^{*} *(p)\right)
$$

The question of what syntactical category $f$ belongs to cannot be answered on the basis of equiform variables. It is the case that the $f$ in $f(p)$ is analogous (in the sense of $T E 22)$ to $\operatorname{Vr}$ in $\operatorname{Vr}(p)$ and hence it is natural to say they are in the same syntactical category. These two remarks motivate

TE32 $[A B C]: . A \varepsilon$ l homosemp $(B C) . \equiv: A \varepsilon A: A \varepsilon \operatorname{frp}(C) . B \varepsilon \operatorname{frp}(C) . v .\left[{ }_{\exists} D\right]$. $D \varepsilon$ ingrthp $(C) . A \varepsilon$ cnvar $(B D) . \vee .\left[{ }_{\exists} D E\right] . D \varepsilon$ ingrthp $(C) . E \varepsilon$ ingrthp $(C)$. $A \varepsilon \operatorname{An}(B D E)$

$A$ is a direct homoseme of $B$ with respect to thesis $C$. This means that $A$ and $B$ are in the same syntactical category.

The following theorems are easily proved.

T4.3.1 $[A B C]: A \varepsilon$ thp $(B) . C \varepsilon$ thp $(B)$.つ. $A \varepsilon$ l homosemp $(C B)$

T4.3.2 $[A B]: A \varepsilon$ thp $(B)$.Ј. $A \varepsilon$ lhomosemp $(A B)$

[TE32, TE31]

T4.3.3 $[A B C]: A \varepsilon$ l homosemp $(C B)$.Ј. $A \varepsilon$ lhomosemp $(A B)$

T4.3.4 $[A B C]: A \varepsilon$ lhomosemp $(B C)$. Ј. $B \varepsilon$ lhomosemp $(A C)$

[TE32, T4.1.37, T4.2.23]

These last two theorems show reflexivity and symmetry, but transitivity is still lacking. The following example shows why

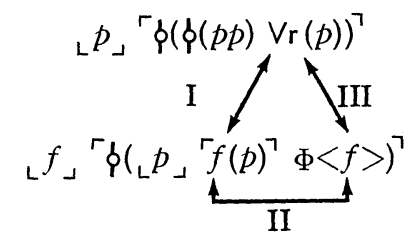


$\mathrm{Vr}$ and $f$ are in the same category since they are analogous functors (arrow I). The two $f$ 's in (9) are in the same category (arrow II) since they are equiform variables. But lhomosemps are connected by analogy or by equiform variables, but not by both. Hence, $\mathrm{Vr}$ and the second $f$ in (9) are not lhomosemps (arrow III).

In order to obtain transitivity, and hence arrow III, we must induct. As we have no recursive definitions we use the Frege-Dedekind technique of ancestral relations. Before giving TE33 let us see how it works. We want $A$ and $B$ to be in the same class iff there is a finite sequence

$$
A, C_{1}, C_{2}, \ldots, C_{\mathrm{n}}, B
$$

such that adjacent terms are lhomosemps. We insure this in the following way. Let $a$ be any class which includes $B$ and which is closed under the relation of thomosemp, i.e.,

$$
[D E]: D \varepsilon a . E \varepsilon \text { l homosemp.(DC).つ. } E \varepsilon a
$$

(this is the condition which guarantees transitivity). Now take the smallest class which satisfies these properties. (For a simpler example of how this works see Leśniewski [20]).

Finally we remark that the clause

$$
[D]: D \varepsilon a . \supset . D \varepsilon \text { l homosemp }(D C)
$$

is included in TE33 so that the intersection is finite. This is true since we consider only $a$ 's which are subsets of lhomosemp $(D C)$. These sets are finite (for any given $D$ and $C$ ) since, at any given time, Protothetic contains only a finite number of theses with respect to $B$ and each of these contains only a finite number of expressions and hence lhomosemps. If we were willing to take an unrestricted quantification we could drop this part of the T.E.

TE33

$$
\begin{aligned}
& {[A B C]:: A \varepsilon \text { homosemp }(B C) . \equiv: A \varepsilon A .} \\
& B \varepsilon 1 \text { homosemp }(B C): . \\
& {[a]: . B \varepsilon a:[D]: D \varepsilon a \text {. } . D \varepsilon \text { l homosemp }(D C):[D E]: D \varepsilon a .} \\
& \quad E \varepsilon \text { l homosemp }(D C) \text {. } . \text {. } E \varepsilon a: \supset . A \varepsilon a
\end{aligned}
$$

$A$ is of the same syntactical category as $B$ with respect to thesis $C$.

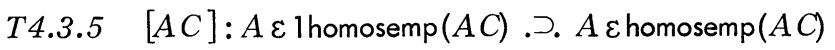

We shall now prove transitivity.

T4.3.6 $[A B C D a]:: A \varepsilon$ homosemp $(B C) . B \varepsilon$ homosemp $(D C) . D \varepsilon a:[E]: E \varepsilon a$. . $E \varepsilon$ l homosemp $(E C):[E F]: E \varepsilon a . F \varepsilon$ l homosemp $(E C)$. $. F \varepsilon a: . \supset . A \varepsilon a$. Hyp(5) .つ:•:

6. $[a]: . D \varepsilon a:[E]: E \varepsilon a . \supset . E \varepsilon$ lhomosemp $(E C):[E F] . E \varepsilon a$. $F \varepsilon$ lhomosemp $(E C)$. $. F \varepsilon a: . \supset . B \varepsilon a:$.

$[T E 33,2]$

7. $B \varepsilon a$.

$A \varepsilon a$

$[6,3,4,5]$

$[1, T E 33,7,4,5]$

T4.3.7 $[A B C D]: A \varepsilon$ homosemp $(B C) . B \varepsilon$ homosemp $(D C)$.Ј. $A \varepsilon$ homosemp $(D C)$ 
4 Special Terms (TE34-45). In this section we give several very technical Terminological Explanations. They are designed for special uses in the next section and consequently we shall discuss them in the context that they will be used.

$T E 34 \quad[A B C D]: . C \varepsilon \operatorname{Antrm}(D A B) . \equiv: C \varepsilon C: C \varepsilon 1 \operatorname{trm}(A) . D \varepsilon \operatorname{ltrm}(B) . v$.

$$
C \varepsilon 2 \operatorname{trm}(A) . D \varepsilon 2 \operatorname{trm}(A)
$$

This is used only in $T E 47$, the rule for distribution of quantifiers. This gives us a method to relate analogous terms of generalizations where the essence is an equivalence. The following diagram may be useful.

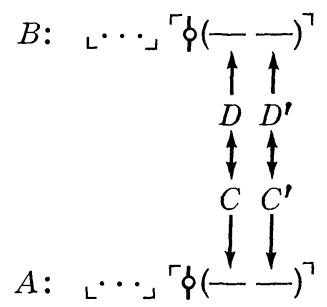

$T E 35 \quad[C D E a]: D \varepsilon \operatorname{Corr}(E C a) . \equiv$

$D \varepsilon D$.

$E \varepsilon a$.

$(a \cap \operatorname{prcd}(E)) \infty(\operatorname{int}(\operatorname{Sbqntf}(C)) \cap \operatorname{prcd}(D))$

This correspondence is used only in formulating the rule of substitution ( $c f$. TE49 below). Taking advantage of some knowledge from TE49 we can say that Corr will be used only in the following situation

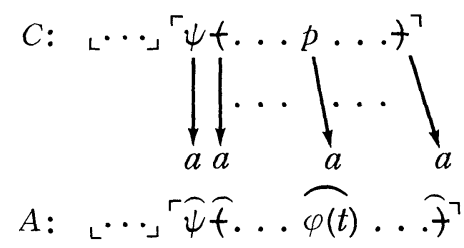

where $D$ is one of the words in the essence of $C$ and $E$ is one of the $a$ 's in $A$, in particular $E$ is that $a$ with the same number of $a$ 's preceding as $D$ has words of Essnt $(C)$ preceding.

TE36 $[A B]: . A \varepsilon \operatorname{fnctgen}(B) . \equiv$ :

$A \varepsilon$ fnct .

$\operatorname{prntm}(A) \leq \operatorname{prntm}(B):$

$[C D]: C \varepsilon \operatorname{prntm}(A) . D \varepsilon \operatorname{prntm}(B) .(\operatorname{prntm}(A) \cap \operatorname{scd}(C)) \infty(\operatorname{prntm}(B) \cap$ $\operatorname{scd}(D))$..$C \varepsilon$ simprntm $(D)$

$A$ is a function generated by function $B$ iff $A$ has fewer links than $B$ and each link of $A$ is similar to the corresponding link in $B$. For example if $B$ is

$$
\Phi \neq p \neq+q+(r)
$$

then it generates the following functions 


$$
* \neq p \neq+q+(r), \psi+q \succ(s), \Phi(\llcorner u\lrcorner\ulcorner u\urcorner)
$$

but none of

$$
\oint \neq p \neq \neq \neq q \neq+r+(s), \oint(p q), \oplus+p q+(r) .
$$

This T.E. will be of great use in formulating the directive concerning substitution: The only functions which can be substituted are those which are generated by functions already in the system. We state without proof.

T4.4.1 $[A]: A \varepsilon$ fnct . $\supset . A \varepsilon$ fnctgen $(A)$

$T E 37 \quad[A B C D E]:: C \varepsilon$ constp $(B A D E) . \equiv:$.

$D$ E homosemp $(E B)$.

$C \varepsilon \operatorname{cnf}(D)$ :

$[F]: F \varepsilon$ ingrthp $(B) . \supset . D \varepsilon \sim[\operatorname{var}(F)]:$

$\left[{ }_{\exists} F G\right] . F \varepsilon \operatorname{ingr}(A) . G \varepsilon \operatorname{ingrthp}(B) . C \varepsilon \operatorname{An}(E F G)$

$T E 38[A B C]: . C \varepsilon$ constp $(B A) . \equiv . C \varepsilon C .\left[{ }_{\exists} D E\right] . C \varepsilon$ constp $(B A D E)$

$C$ is a constant of Protothetic with respect to thesis $B$ and (occurring in) $A$. We now state without proof.

\section{T4.4.2 $[A B C]: C \varepsilon$ constp $(B A)$.D. $C \varepsilon \operatorname{vrb}(A)$}

This term is used only three times, in TE46.3, 4, and TE49.8. TE37.3 states that no word equiform to a constant $C$ has ever served as a variable. Thus, once we quantify a letter it may never be used as a constant. Condition TE 37.4 guarantees that $C$ is being used properly (if a functor, the right type of parentheme follows; if a variable, it occupies a spot which can hold something of that type) in expression $A$, which we intend to add as a thesis after $B$.

TE39 $[A B C D E F]: . A \varepsilon q$-homosemp $(B C D E F) . \equiv$.

$E \varepsilon$ homosemp $(F C) . A \varepsilon A$.

$[{ } G I] . G \varepsilon \operatorname{ingr}(D) . I \varepsilon$ ingrthp $(C) . A \varepsilon \operatorname{An}(E G I)$

$\left[{ }_{[} G I\right]: G \varepsilon$ ingr $(D) . I \varepsilon$ ingrthp $(C) . B \varepsilon$ An $(F G I)$

$T E 40 \quad[A B D F]: . F \varepsilon q$-homosemp $(D B A) . \equiv F \varepsilon F$.

$\left[{ }_{\exists} G H\right] . F \&$ q-homosemp $(D B A G H)$

It is meaningful to say two expressions are of the same syntactical category only when they are ingredients of theses of Protothetic. Hence, when we are considering an inscription $A$ as a possible thesis, it is not meaningful to say certain parts of it are in the same syntactical category. The term q-homosemp is used to circumvent this difficulty. We say $F$ and $D$ will be of the same syntactical category when $A$ is added as a thesis after $B$ if $F$ and $D$ are analogous to ingredients of theses which are already of the same syntactical category.

$T E 41 \quad[A B C D E] \ldots C$ efnctp $(B A D E) . \equiv$ :

$D$ E homosemp $(E B)$.

$C \varepsilon$ fnctgen $(D)$ :

$\left[{ }_{3} F G\right] . F \varepsilon \operatorname{ingr}(A) . G \varepsilon \operatorname{ingrthp}(B) . C \varepsilon \operatorname{An}(E F G)$

$T E 42 \quad[A B F G H I] .: F \varepsilon \operatorname{varp}(G B A H I) . \equiv$ : 


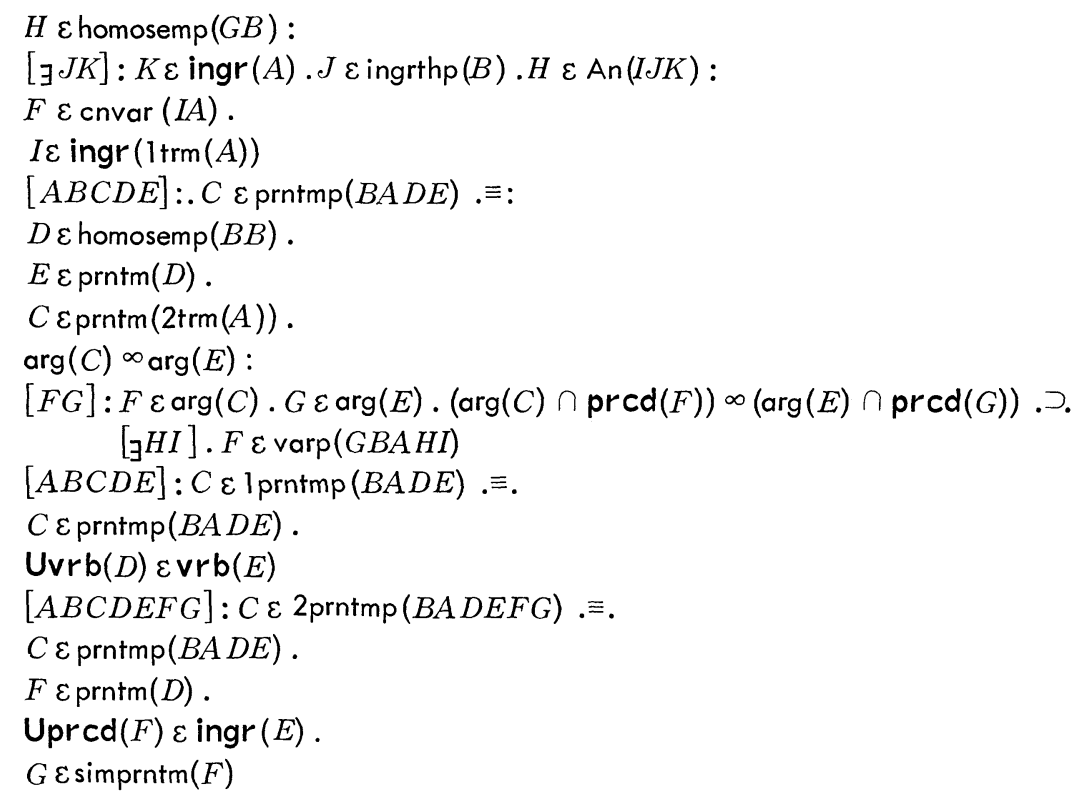

These technical terms will be useful for choosing parentheses in definitions. They will be explained later when used.

5 The T.E.'s for Definition, Quantification, Detachment, Substitution and Extensionality (TE46-51). The previous forty-five Terminological Explantions were all in preparation for this section which is the key to Protothetic.

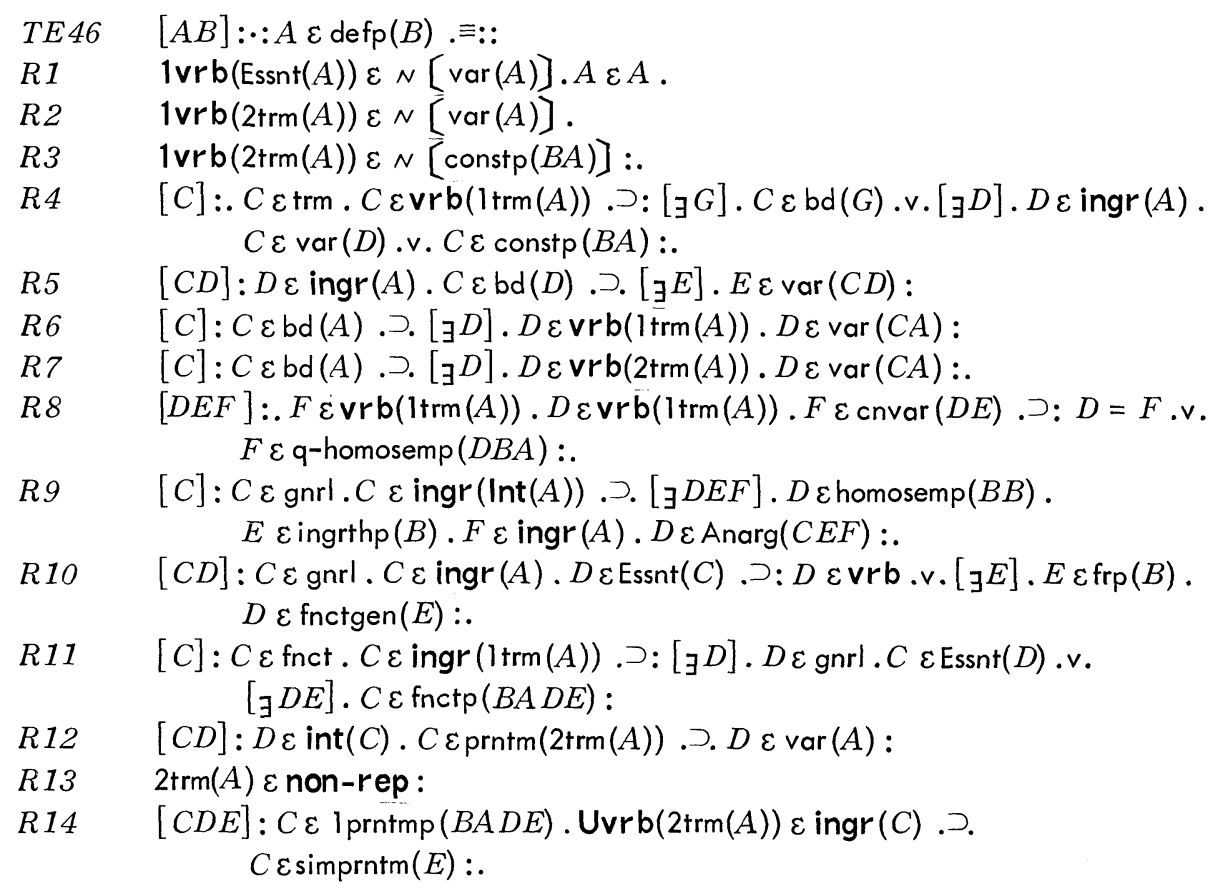




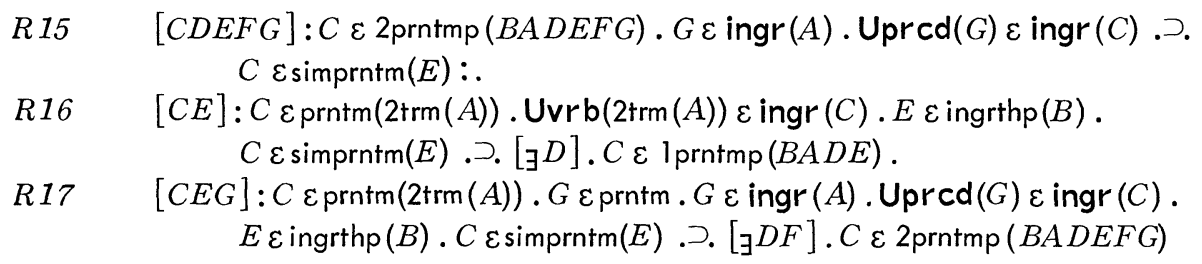

$A$ is a definition after thesis $B$.

Several conjuncts of TE46 apply to any prospective thesis of Protothetic. They are $T E 46.4,5,8,9,10,11$. Let us ignore them for the moment and concentrate on the conjuncts peculiar to definitions.

First remark that a definition need not be a generalization. But its essence, by $T E 46.2$, must be an equivalence. The second term of $A$ can contain no variables in this case (TE46.3) and is thus a term. In this case then we are defining a constant without any parameters, or, as one usually says, a constant. The definition thus has the form

$$
\phi(\psi *)
$$

where $*$ is the newly defined constant and $\psi$ is a well formed formula (the other conjuncts guarantee this) which contains no free variables.

As examples of this type of definition consider the following definitions of 0 and 1 (falsehood and truth)

$$
\begin{aligned}
& \phi\left(\left\llcorner u_{\lrcorner}\ulcorner u\urcorner 0\right)\right. \\
& \phi\left(\phi\left(\left\llcorner{ }_{\lrcorner}\left\ulcorner u^{\urcorner}{ }_{\llcorner} u\right\lrcorner\ulcorner u\urcorner\right) 1\right)\right.
\end{aligned}
$$

These are not the only definitions of constants possible, but any constant defined will be equivalent to either 0 or 1 .

Now we move to the more interesting case of where $A$ is a generalization. From $T E 46.2,5,13$ we can prove $2 \operatorname{trm}(A) \varepsilon$ fnct and so $A$ has the form

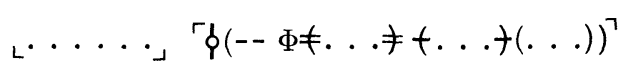

The first term of $A$, since it is an argument, is either a term (TE1), generalization, or function. All three cases can occur but if the definiens is a term then the definiendum has the shape

$$
\operatorname{As}(x)
$$

i.e., it has only one parameter. The definition is precisely (up to change of symbolism)

$$
\llcorner x\lrcorner\lceil\zeta(x \operatorname{As}(x)),
$$

which is usually called Assertium.

TE46.12 tells us that the words in the parenthemes of the function which is the definiendum are variables. Hence the parenthemes of the definiendum $(2 \operatorname{trm}(A))$ look like

$$
<p q \ldots s\rangle
$$

and the definiendum looks like 


$$
\Phi \neq p q \ldots s \neq+f g \ldots+\ldots(u v \ldots)
$$

where all the symbols are different (TE46.13).

TE46.6, 7 tell us that every binder in $A$ binds at least one term in the definiens and exactly one variable in the definiendum. These conditions are necessary to prevent contradictions ( $C f$. eg., Carnap [3]).

Combining all of this information we see that definitions have the form

${ }_{\llcorner} p q f g u v \ldots .\left\ulcorner\phi(\psi(p q f g u v \ldots) \Phi \neq p q \ldots \neq+f g \ldots+\ldots(u v \ldots))^{\top}\right.$

It remains to show how TE46.14-17 govern the choice of parentheses in the definiendum. These conjuncts are very important for they insure that the syntactical categories are uniquely determined on syntactical grounds. In particular, if we know 1) the shape of the parentheses and 2) the number of arguments of a parentheme, then we know the syntactical category of each argument and also the syntactical category of the functor which precedes this parentheme.

Conditions 14 and 15 force us to use certain parentheses; 16 and 17 prevent us from using others. Conditions 14 and 16 govern the choice of parentheses for the last (i.e., rightmost) parentheme in the definiendum. Conditions 15 and 17 govern the choice of the other parentheses in the definiendum in a manner akin to inductive definitions, but of course without using natural numbers.

The explanations of conditions 14 and 16 which follow refer to the following diagram:

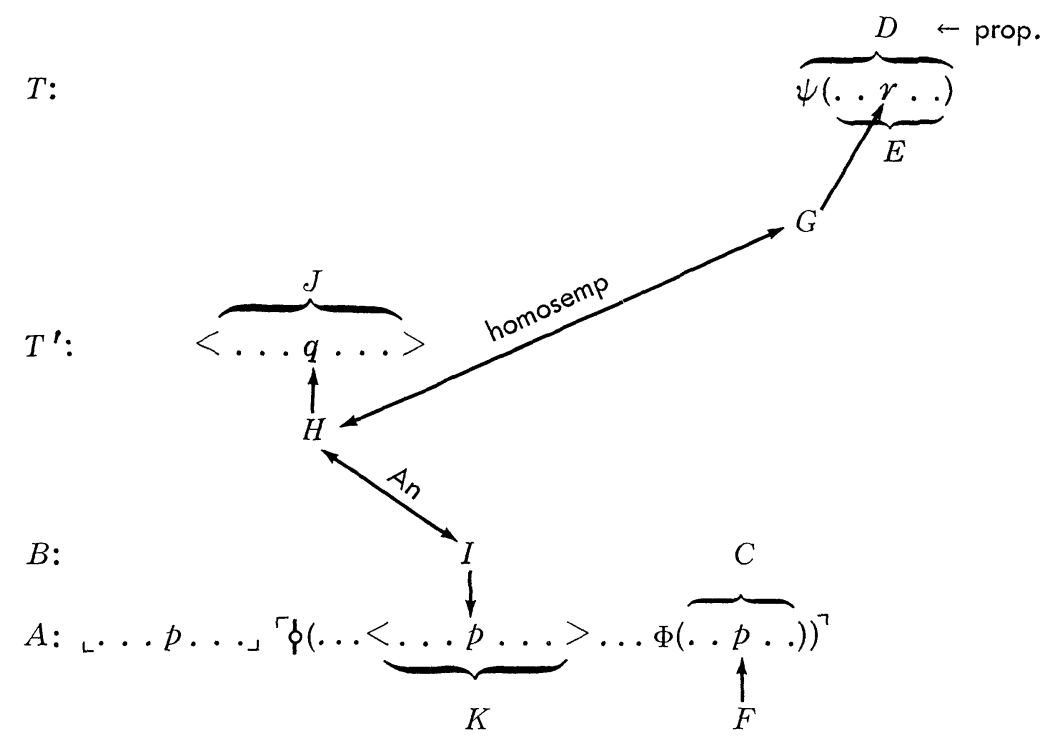

Condition 14 dictates that whenever there is a parentheme $E$, which is the last parentheme of a function $D$, which is in the syntactical category of propositions, and when corresponding arguments of $E$ and $C$, which is the last parentheme of the definiendum (i.e., 2trm) of $A$, are destined to be in 
the same category, then one must use the same kind of parentheses for $C$ as $E$ has.

We speak of an argument $F$ of $C$ as being "destined to be" in a certain syntactical category since inscription $A$, of which $C$ is a part, is not yet a thesis of Protothetic and accordingly its part $F$ cannot be in any syntactical category. What we intend is that if and when $A$ is added to Protothetic as a new thesis according to the rule of definition then $F$ will be in the same syntactical category as $E$.

The category to which $F$ is destined to belong is established as follows: Relate $F$ back to an equiform variable in the definiens, say $I$, which is in parentheme $K$ (if $I$ is a functor the argument is similar). Then $I$ can be related to the variable $H$ in parentheme $J$ which is part of some thesis of Protothetic with respect to $B$. This is done in such a way that $I$ and $H$ are analagous arguments (or functors) in $K$ and $J$ respectively. Now since $H$ and $G$ are both ingredients of theses of Protothetic it is meaningful to say that they are in the same syntactical category. Since $H$ and $I$ are analagous they should be in the same category, and since $I$ and $F$ are equiform variables they should be in the same category. Finally, by transitivity, $F$ and $G$ are destined to be in the same category.

Condition 16 states that when the last parentheme $C$ of the definiendum is similar to the last parentheme $E$ of a function $D$ which is in the syntactical category of propositions then corresponding arguments of the parenthemes $C$ and $E$ are destined to belong to the same syntactical category. In particular this says that if the nth argument of $E$ is of different category than the nth argument of $C$ then the parenthemes $E$ and $C$ are not similar, i.e., either $C$ and $E$ have different numbers of arguments or they have non-equiform parentheses.

The following examples show how these two conditions govern the choice of parentheses in definitions.

The axiom contains only parentheses of the shape (--) and these are preceded by a functor in the category S/SS. Condition 14 dictates that in any definition where we have a sentential function of two sentential variables then the arguments must be enclosed in parentheses equiform to $(--)$. For example, in

(1) $\left.\left.{ }_{\llcorner} p q\right\lrcorner{ }_{\lrcorner} \phi\left(\phi(q p) \phi_{1}(p q)\right)\right\urcorner$

the choice of parentheses in the definiendum is forced by condition 14 since the arguments of $\phi_{1}$ are destined to be in the category $S$, and there are two of them. Any other choice of parentheses would violate 14 .

(2) $\left.{ }_{\llcorner} p q r\right\lrcorner\left\ulcorner\phi\left(\phi(p \phi(q r)) *_{1}+p+(q r)\right)\right\urcorner$

Condition 14 forces the choice of the last parentheses of (2). But any type of parentheses, except (-), could be used for the second parentheme. Condition 14 is satisfied for the following since its hypothesis is false:

(3) $\left.\left.{ }_{\llcorner} p\right\lrcorner{ }^{\top} \phi\left(\phi(p p) *_{2}+p t\right)\right)^{\top}$

However, this is unacceptable as a definition since the only parentheme in 
the definiendum is similar to the second parentheme in the definiendum of (2), which is not the last parentheme of (2) as is required by condition 16 . The following is eligible as a definition immediately after (2):

(4) $\left.{ }_{L} p\right\lrcorner{ }_{\lrcorner}(\phi(p p) \vee \mathrm{r}(p))$

This satisfies both 14 and 16 vacuously, since there is no one argument parentheme in the system which is last in its definiendum. Actually any choice of parentheses, except $t-t$, could be made here. However, in all further definitions where the last parentheme contains but one argument and that of the syntactical category of propositions, we must use parentheses equiform to those in (4). The freedom to choose the type of parenthesis in (4) enables one to display analogy between defined terms through the notation.

Both conditions 14 and 16 are necessary to keep the syntactical categories separate. If we dropped 14 then we could add

(5) $\left.{ }_{L} p\right\lrcorner{ }^{\top} \phi\left(\phi(p p) *_{3}<p>\right)^{\top}$

and thus get the erroneous impression that $\mathrm{Vr}$ and $*_{3}$ belong to different categories. If we dropped 16 then we could add

(6) $\left.\left.{ }_{\llcorner} f p\right\lrcorner{ }^{\circ} \phi\left(f(p) *_{4}(f p)\right)\right\urcorner$

which would give the impression that the functor $*_{4}$, which should be of the category $\mathrm{S} /(\mathrm{S} / \mathrm{S}) \mathrm{S}$, is of category $\mathrm{S} / \mathrm{SS}$. Thus if 16 were omitted the categories would coalese while if 14 were dropped the categories would multiply needlessly.

Conditions 15 and 17 govern the choice of parentheses for all but the last parentheme of the definiendum. The explanations which follow refer to the following diagram:

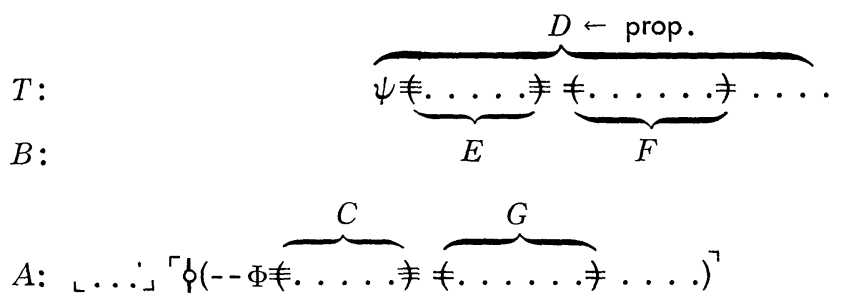

Condition 15 dictates that parenthemes $C$ and $E$ will have the same type of parentheses when they have the same number of arguments, when corresponding arguments are destined to be in the same syntactical category, and when the parenthemes following them (i.e., $G$ and $F$ respectively) are similar.

Conversly condition 17 stipulates that when the parenthemes $C$ and $E$ are similar and when $C$ is followed by a parentheme $G$ then there is a parentheme $F$ following $E$ which is similar to $G$ and moreover $C$ and $E$ have the same number of arguments and corresponding arguments are destined to be in the same category.

These conditions are very similar to 14 and 16 respectively and so we 
go right to examples. The following is a legitimate definition immediately after (1), (2), and (4) above:

(7) ${ }_{\llcorner} p q r_{\lrcorner}\left\ulcorner\phi\left(\phi(\phi(p q) r) *_{5}+p+(q r)\right)\right\urcorner$

To use another shape of parentheses on the second parentheme would violate condition 15. This arrangement of parentheses was used in (2) and must always be used when a functor of type $(\mathrm{S} / \mathrm{SS}) / \mathrm{S}$ is involved. We can also add the definition

(8) $\left.\left.{ }_{\llcorner} p q r s\right\lrcorner \gamma\left(\phi(\phi(p q) \phi(r s)) *_{6}+p q+(r s)\right)\right\urcorner$

The use of the same shaped parentheses for the second parenthemes of (7) and (8) causes no confusion as these parenthemes contain different numbers of arguments. Both conditions 15 and 17 are satisfied as their hypotheses are false.

(9) $\left.\left.{ }_{\llcorner} p q r\right\lrcorner \Gamma \phi\left(\phi(p \phi(q r)) *_{7}+p q+(r)\right)\right\urcorner$

This violates condition 17 since the second parenthemes of (8) and (9) are similar but the first parentheme of (8) is not similar to the first of (9). So a different choice of parentheses must be made for the second parentheme of (9).

Condition 17 prevents us from taking

$$
\left.\left.{ }_{\llcorner} p q\right\lrcorner\left\ulcorner\phi(\llcorner f\lrcorner f(p q)\urcorner *_{8}(p q)<f>\right)\right\urcorner
$$

as a definition since the second parentheme of (10) is similar to the first parentheme of (2), whereas condition 17 requires that if the second parentheme of a proposed definition is similar to any other parentheme then that parentheme, among other things, must be followed by another parentheme. In particular 17 prevents us from having both $+--+($.$) and$ (. .) $t--t$ in the system.

Finally let us consider the inscription

$$
{ }_{\llcorner} p q r_{\lrcorner}\left\ulcorner\phi\left(\phi(p \phi(q r)) *_{9}(p)(q r)\right)\right\urcorner
$$

This satisfies conditions $14-17$, but does not satisfy 13 since the would-be definiendum contains two equiform symbols. Thus it is not eligible as a definition of Protothetic. Sobociński has remarked (orally) that Leśniewski would not have allowed this as a definition either. However (11) does satisfy Leśniewski's formulation of the rule of definition as formulated in [18]. Thus it seems that Leśniewski's verbal statement of the rule does not agree with his printed one. This is the only point at which our rule differs from that given by Leśniewski in [18], and this point does not affect the deductive strength of the theory, but only the shape of parentheses in some theses.

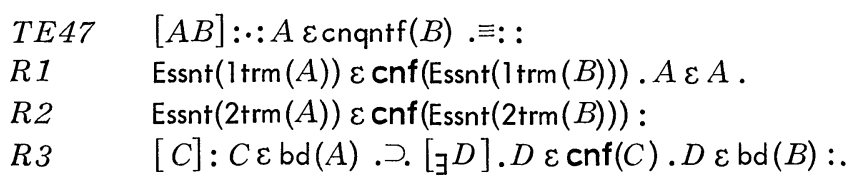




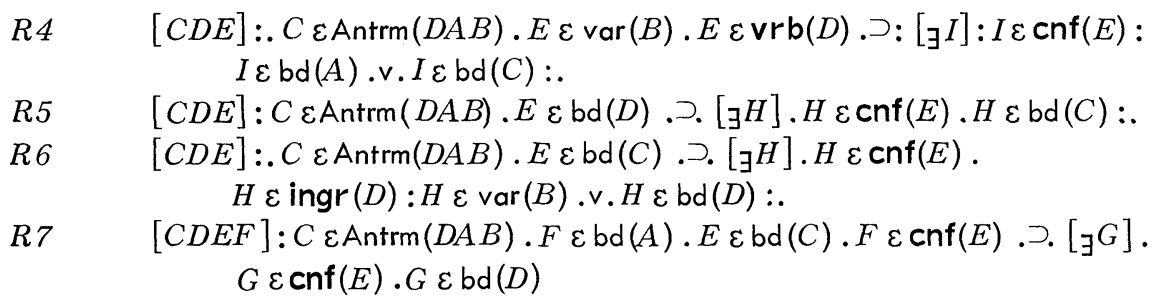

$A$ is a consequence of $B$ by quantification. TE47.1, 2 tell us that both $A$ and $B$ are generalizations (admittedly both $A$ and $B$ could be equivalences but this case is both trivial and uninteresting since we are trying to distribute quantifiers), and moreover that they are generalizations of equivalences. Also corresponding terms have equiform essences. Let us extend the schematic diagram following TE34:

$B:$

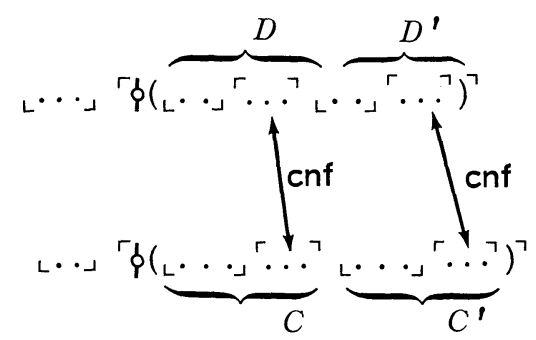

$T E 47.3$ says any binder in $A$ is equiform to a binder in $B$. Semantically this means any binder which was not distributed is a binder in both $A$ and $B$.

$T E 47.4$ says that for every variable in $B$ which occurs in $D\left(D^{\prime}\right)$ there is a binder in $A$ or $C\left(C^{\prime}\right)$ which is equiform to that variable. This notation is quite cumbersome so we introduce the following.

We say the binders of $A$ are an equiformity subset of the binders of $B$ iff for every binder of $A$ there is an equiform binder of $B$. Then TE47.3 merely says that the binders of $A$ are an equiformity subset of the binders of $B$.

TE47.5 says that if $C\left(C^{\prime}\right)$ is an analogous term to $D\left(D^{\prime}\right)$ in $A$ and $B$ respectively then the binders of $D$ are an equiformity subset of the binders of $C$.

TE47.6 says any binder in $C$ has an equiform binder in $D$ or there is some occurrence of that binder in $D$ which is bound in $B$.

Finally TE47.7 says that if the quantifiers of $A$ and $C$ contain equiform words then there is a word in the quantifier of $D$ equiform to it.

Let us give several examples. If $B$ is

$$
\left.{ }_{\llcorner} p q\right\lrcorner\ulcorner\phi(\phi(p q) \phi(q p))\urcorner
$$

then $A$ could be $B$ or

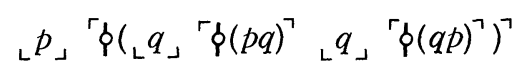

or a similar expression with $p$ distributed or we could distribute both variables and get

$$
\left.\phi\left(\left\llcorner_{\llcorner} p q,\ulcorner\phi(p q)\urcorner,{ }_{\llcorner} p q\right\lrcorner \Gamma^{\top} \phi(q p)\right\urcorner\right)
$$


In this case the order of the binders in either quantifier could be altered. As a more complicated example consider

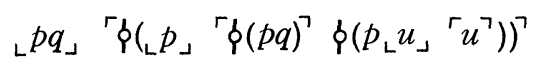

Then $A$ could be, if we distribute $p$

$$
{ }_{\llcorner} q_{\lrcorner}\left\ulcorner\phi\left({ }_{\llcorner} p_{\lrcorner} \Gamma \phi(p q){ }^{\urcorner}{ }_{\llcorner} p_{\lrcorner} \Gamma\left(p_{\llcorner} u\right\lrcorner\left\ulcorner u^{\urcorner}\right)^{\urcorner}\right)\right\urcorner
$$

or, if we distribute $q$

$$
\left.{ }_{\llcorner} p\right\lrcorner \Gamma_{\zeta}\left({ }_{\llcorner} p q,\ulcorner\phi(p q)\urcorner \phi\left(p_{\llcorner} u_{\lrcorner}\ulcorner u\urcorner\right)\right) .
$$

$T E 48 \quad[A B C]: A \varepsilon \operatorname{cnequl}(B C) \equiv . C \varepsilon \operatorname{cnf}($ leqvl $(B))$.

$$
A \varepsilon \operatorname{cnf}(2 \operatorname{eqvl}(B))
$$

$A$ is the result of detaching $C$ from $B$.

Schematically we have $B$ :

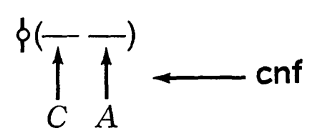

N.B. We are not considering detachment under quantifiers, i.e., we do not want the rule

$$
\begin{aligned}
& \left\llcorner. .\left\ulcorner C^{\urcorner}\right.\right. \\
& \frac{\llcorner. .\lrcorner \phi(C A)^{\urcorner}}{\llcorner. .\lrcorner A^{\urcorner}}
\end{aligned}
$$

But the rule

$$
\frac{\begin{array}{c}
C \\
\phi(C A)
\end{array}}{A}
$$

TE49 $[A B C a]: \cdot: A \varepsilon$ cnsbst $(B C a) . \equiv::$

$R 1 \quad \operatorname{Essnt}(A) \& \operatorname{Cmpl}(a) . A \& A$.

R2 $\quad a \infty \operatorname{int}(\operatorname{Sbqntf}(C)):$.

$R 3 \quad[D E]: . D \varepsilon \operatorname{int}(\operatorname{Sbqntf}(C)) . D \varepsilon \operatorname{Corr}(E C a) . \supset: D \varepsilon \operatorname{var}(C) . \vee . D \varepsilon \operatorname{enf}(E):$.

$R 4 \quad[D E]: D \varepsilon \operatorname{int}(\operatorname{Sbqntf}(C)) . D \varepsilon \operatorname{Corr}(E C a)$. .

$E \varepsilon$ trm $\cup$ gnrl $\cup$ fnct $\cup \operatorname{cnf}(D):$.

$R 5 \quad[D E F G]: D \varepsilon \operatorname{cnvar}(E C) . D \varepsilon \operatorname{Corr}(F C a) . E \varepsilon \operatorname{Corr}(G C a) . \supset . F \varepsilon \operatorname{cnf}(G):$.

$R 6 \quad[D E F G H I K L]: D \varepsilon \operatorname{ingr}(\operatorname{Essnt}(C)) . E \varepsilon b d(D) . F \varepsilon \operatorname{var}(K C) . F \varepsilon \operatorname{ingr}(D)$. $E \varepsilon \operatorname{Corr}(G C a) . F \varepsilon \operatorname{Corr}(H C a) . L \varepsilon \operatorname{ingr}(A) . I \varepsilon \operatorname{var}(G L)$.. $I \varepsilon \sim[\operatorname{ingr}(H)]:$.

$R 7 \quad[D E]: . D \varepsilon$ bd $(A) . E \varepsilon \operatorname{cnf}(D) . E \varepsilon \operatorname{ingr}(C) . \supset:\left[{ }_{\exists} F\right] . E \varepsilon \operatorname{bd}(F) . v$. $[\exists F G] . F \varepsilon \operatorname{ingr}(C) . E \varepsilon \operatorname{var}(G F):$.

$R 8 \quad[D]: . D \varepsilon \operatorname{trm} . D \varepsilon \operatorname{vrb}(A) . \supset:\left[{ }_{\exists} G\right] . D \varepsilon$ bd $(G) \cdot \vee \cdot\left[{ }_{\exists} G\right] . D \varepsilon \operatorname{var}(G)$. $G \varepsilon \operatorname{ingr}(A)$.v. $D \varepsilon$ constp $(B A):$.

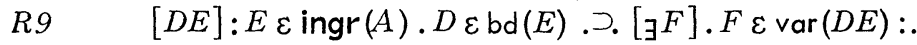

$R 10 \quad[D E F]: . E \varepsilon \operatorname{ingr}(A) . F \varepsilon$ cnvar $(D E) . \supset: F=D$.v. $F \varepsilon q$-homosemp $(D B A)$ :. 


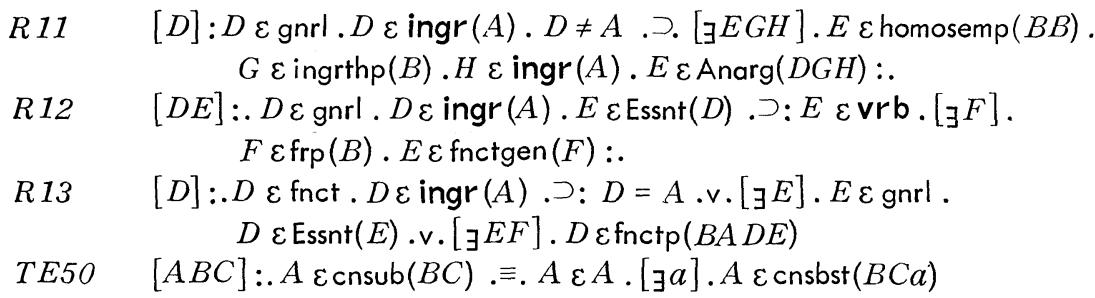

$A$ is a consequence of substitution in $C$ with respect to thesis $B$.

Contrary to the commonly held opinion that Hilbert and Bernays were the first (in 1934) to correctly state the rule of substitution for functional variables it should be noted that Leśniewski had already done it in [18] in 1929. Leśniewski cleverly solves the problem of substitution by not saying what is substituted for the variables. This paradoxical statement will be explained by means of an example. Suppose we have

$$
\llcorner p q r\lrcorner\ulcorner(\varphi(r q p) p)\urcorner
$$

and wish to substitute $\operatorname{Vr}(p)$ for $p$. Everyone knows that we get

$$
\text { ¿ } p q r,\ulcorner\phi(\varphi(r q \vee r(p)) \vee r(p))\urcorner .
$$

We do not care how one divines this but only need give a method to verify that it is correct. Look at the essence of (13) and break it up into 10 parts (= the number of words in the subquantifier of (12)) in such a way that constants, corners, and parentheses in (12) correspond to equiform words in (13) and also so that variables in (12) correspond to expressions in (13). This nearly forces us to view (13) in the following way

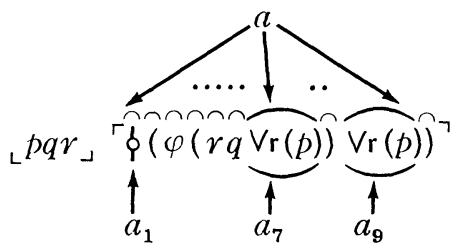

These ten expressions constitute $a$. The argument $D \varepsilon$ Corr $(E C a)$ used in TE49.3, 4, 5, 6 corresponds words $D$ in the subquantifier of $C$ (i.e., (12) in our example) to expressions $E \varepsilon a$. TE 49.3 says corresponding things are equiform except (possibly) in the case when $D$ is a variable in $C$. The only places which are not equiform are those for which we substituted. Naturally enough the $a$ 's all must be terms, generalizations or functions, or equiform to that to which they correspond. Semantically speaking this says we can only substitute terms, generalizations or functions ( $c f . T E 17)$.

TE49.5 guarantees that we substitute equiform expressions for equiform variables in $C$. This requirement is so natural as to defy comment.

TE 49.6 is the famous and troublesome clause which states that interior quantifiers may never trap variables. If we have

$$
\llcorner p q\lrcorner\ulcorner\psi(\llcorner p,\ulcorner\phi(p q)\urcorner p)\urcorner
$$

then we may not substitute $p$ for $q$ to get 


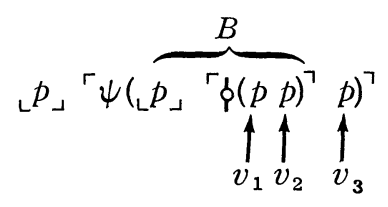

for then we have both $v_{1}$ and $v_{2}$ bound in $B$ whereas we wanted $v_{2}$ to be bound by the MGQ and hence be an equiform variable to $v_{3}$.

The remaining conjuncts of TE49 concern arbitrary theses and will be discussed at the end of this section.

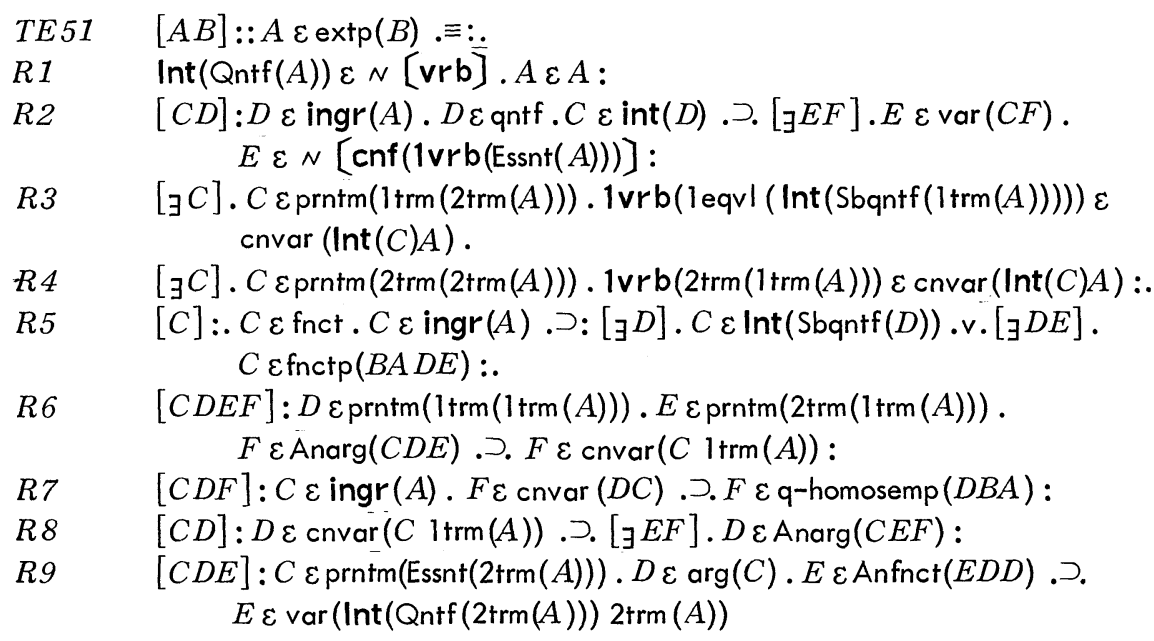

$A$ is a thesis of extensionality with respect to thesis $B$.

Let us begin with an example of a thesis of extensionality:

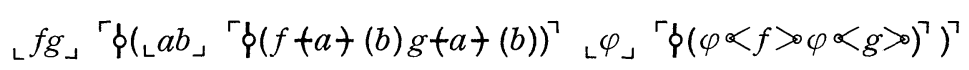

and examine what the rule tells us about it.

$T E 51.1$ says that $A$ is a generalization containing at least two binders. From TE51.3, especially from

$$
C \varepsilon \operatorname{prntm}(1 \operatorname{trm}(2 \operatorname{trm}(A)))
$$

we have that the essence of $A$ is an equivalence and that the 2 trm of this equivalence has an essence which is an equivalence. The rest of this conjunct tells us that $\operatorname{lnt}(C)$ is a variable in $A$ and hence a word. Thus the $2 \operatorname{trm}(A)$ has the shape

$$
\text { L_」 } \zeta(-\alpha f>0-\alpha g>0)\urcorner
$$

TE5 1.9 implies that the three dashes represent the same variable $(\varphi)$.

TE51.6 and 8 tell us that the terms of the first term of $A$ are equiform except for the first letter of each term.

The remaining conditions, TE51.5 and 7, apply to any thesis.

Finally we mention several common conjuncts of the T.E.'s which are requirements we want any thesis to satisfy.

TE46.4, TE49.8: Terms are binders, variables or constants. 
TE 46.5, TE49.9: Binders actually bind variables. Hence every quantifier is part of a generalization.

TE 46.8, TE49.10, TE51.7: This guarantees that equiform variables of inscriptions considered for addition to Protothetic as new theses will be in the same syntactical category.

TE46.9, TE49.11: Generalizations occupy argument places which are capable of having arguments from the category of propositions.

$T E 46.10, T E 49.12$ : The essences of generalizations are either words or functions which have been generated by functions occurring in a previous thesis of Protothetic (this prevents substitution from introducing new syntactical categories).

6 The rule of Protothetic:

Under the assumption that $B$ is the last thesis of this system of Protothetic one may add the expression $A$ as a new thesis of Protothetic provided one of the following conditions is fulfilled:

1) $\quad A \varepsilon \operatorname{defp}(B)$.

2) $\quad\left[{ }_{\exists} C\right] . C \varepsilon \operatorname{thp}(B) \cdot A \varepsilon \operatorname{cnqntf}(C)$.

3) $\quad\left[{ }_{\exists} C D\right] . C \varepsilon$ thp $(B) . D \varepsilon$ thp $(B) \cdot A \varepsilon$ Cnequl $(C D)$.

4) $\quad[\exists C] . C \varepsilon \operatorname{thp}(B) . A \varepsilon \mathrm{cnsub}(B C)$.

5) $A \varepsilon \operatorname{extp}(B)$.

\section{CHAPTER V}

In this chapter we sketch the proof that our formulation of the rule of Protothetic has the same deductive strength as that which Leśniewski presented in [18], pp. 63-76. The reader should have [18] at hand.

1 Definability of Lesniewski's primitive terms in MP. The first step toward proving equivalence of our rule and Leśniewski's is to show that his primitive terms are definable in system MP. The definitions seem obvious; but there are subtle difficulties involved. To bring these out we describe again how the rule of Protothetic was formulated.

Leśniewski formulated the rule by giving a number of Terminological Explanations which prescribed exactly what he meant by detachment, substitution, etc. He realized that his T.E.'s did not belong to the system itself, and so it was his practice-one fortunately thwarted in [18] by space limitations-to express the T.E.'s in ordinary language. Hence it is understandable that the undefined terms which he used to express the T.E.'s were explained by only a few words of commentary. It is here that our difficulty lies-for we have no way of being certain that our definitions of his primitive terms agree with the intuitive meanings that he attached to them.

What we do is to give definitions in MP of Leśniewski's undefined terms and then argue intuitively that we have captured the meanings which he intended. We shall use two devices for this:

a) We refer to theorems in $M$ and MP which agree with Leśniewski's commentary on his undefined terms. 
b) By examining Leśniewski's T.E.'s we deduce certain properties of his undefined terms which he must have intended if he was to be consistent with the remark ([18], p. 63) that the separate conjuncts of his T.E.'s are logically independent.

The following is a list of Leśniewski's undefined terms:

$\begin{array}{ll}A \varepsilon b & \operatorname{prntsym}(A) \\ \operatorname{Id}(A) & \operatorname{cnf}(A) \\ N[a] & \mathscr{U l} \\ a \cap b \cap \ldots \cap k & \operatorname{thp} \\ a \cup b \cup \ldots \cup k & \operatorname{ingr}(A) \\ a \infty b & \operatorname{prcd}(A) \\ a<b & \operatorname{scd}(A) \\ \operatorname{vrb} & \operatorname{Uprcd}(A) \\ \text { expr } & \operatorname{Uingr}(A) \\ \operatorname{prnt} & \operatorname{lingr}(A) \\ \operatorname{prntl} & 2 \operatorname{ingr}(A)\end{array}$

Of these $\varepsilon$, Id (i.e., $=), N, \cap, \cup, \infty,<$ are terms of Ontology. Their definitions can be found in Chapter $I, 3$.

Of the remaining terms $\mathfrak{A l}$ holds a special place, for it as an individual name, viz., the name of the axiom of Protothetic for which Leśniewski stated his rule $\left(C f_{.},[18]\right.$, p. 59). This axiom is not the same one that we have used. For this reason it will be necessary to adjust Leśniewski's rule to the axiom $\mathfrak{A} r$ which we use. These changes are minor and are necessitated by the different number of binders in the quantifiers of $\mathfrak{A l}$ and $\mathfrak{H} \mathfrak{x}$.

We now proceed to define the rest of these terms in MP.

Df1.1 $[A]: A \varepsilon \mathbf{v r b}_{\mathrm{L}} \equiv . A \varepsilon \mathbf{v r b}$

This definition states that Leśniewski's term $v r b_{L}$ corresponds exactly to our term vrb. We use the subscript $L$ to indicate that the defined term is one of Leśniewski's undefined terms. In support of this definition we mention T2.3.2, T2.4.17, T2.4.18, and T2.4.26 which are the analogues in $M$ (and hence in MP) of Leśniewski's commentary in [18] on his term vrb .

$D f 1.2[A]: A \varepsilon \operatorname{expr}_{\mathrm{L}} \equiv . \equiv \varepsilon \operatorname{expr}$

The justification of defining Leśniewski's notion of expression as equivalent to ours is found in $T 2.4 .2, T 2.4 .3, T 2.4 .4$.

Df1.3 $[A]: A \varepsilon \mathrm{prnt}_{\mathrm{L}} \equiv . A \varepsilon \mathrm{prnt}$

Df1.4 $[A]: A \varepsilon p r n t L_{L} . \equiv . A \varepsilon A .\left[{ }_{\exists} B\right] . B \varepsilon \operatorname{prntm} . A \varepsilon 1 \mathrm{vrb}(B)$

The first of these definitions identifies Leśniewski's notion of parenthesis with ours. The second defines left parenthesis, a term which we eliminated from our system, but can easily define as the first word of a parentheme. Actually the explicit use of left parentheses has been eliminated in our presentation. 


\section{$D f 1.5 \quad[A B]: A \varepsilon \operatorname{prntsym}_{\mathrm{L}}(B) . \equiv . A \varepsilon \operatorname{prntsym}(B)$}

Hence our notion of symmetric parentheses agrees with Leśniewski's.

Up to now there has been little doubt about Leśniewski's intentions; his terms clearly agree with ours. For some of the remaining terms this is not the case and so care must be taken to observe the subscripted L's.

$$
\begin{aligned}
D f 1.6 \quad[A B]: A \varepsilon \operatorname{cnf}_{\mathrm{L}}(B) \equiv . & A \varepsilon \operatorname{enf}(B) . \\
& A \varepsilon \operatorname{expr} . \\
& B \varepsilon \operatorname{expr}
\end{aligned}
$$

Leśniewski's notion of equiformity holds only between expressions, whereas ours is applicable to any inscriptions. He uses $\operatorname{cnf}_{\mathrm{L}}(A)$ as an abbreviation of "mit A gleichgestalteter Ausdruck" and later speaks of "Zwei miteinander gleichgestalteter Ausdrücke" (Cf. [18], p. 60, 62). Together these show that he intends

$$
\begin{aligned}
& {[A B]: A \varepsilon \operatorname{cnf}_{\mathbf{L}}(B) . \supset . A \varepsilon \operatorname{expr} \mathbf{L}} \\
& {[A B]: A \varepsilon \operatorname{cnf}_{\mathbf{L}}(B) . \supset . B \varepsilon \operatorname{expr} \mathbf{L}}
\end{aligned}
$$

Together with $D f 1.2$ these justify the above definition. For further evidence of agreement see T2.4.14, T2.4.15.

Df1.7 $[A]: A \varepsilon$ thp $\mathrm{L}_{\text {. }} \equiv . A \varepsilon$ thp

It should be remarked that whereas Leśniewski took thp as primitive we have taken thp $(B)$. The reasons for this choice were explained in Chapter III, 4.

$$
D f 1.8[A B]: A \varepsilon \operatorname{ingr}_{\mathbf{L}}(B) . \equiv: A \varepsilon A:[C]: C \varepsilon \operatorname{vrb}(A) . \supset . C \varepsilon \operatorname{vrb}(B)
$$

Remembering that our notion of ingredient can hold only between expressions (Cf., T2.4.7, T2.4.8) it is understandable that we can only prove

T5.1.1 $[A B]: A \varepsilon \operatorname{ingr}_{\mathrm{L}}(B) . A \varepsilon \operatorname{expr} . B \varepsilon \operatorname{expr} . \equiv . A \varepsilon \operatorname{ingr}(B) \quad[D 10, D f 1.8]$

Although Leśniewski did not explicitly state it we know he did not intend

$$
[A B]: A \varepsilon \operatorname{ingr}_{\mathbf{L}}(B) \text {. } \supset . B \varepsilon \operatorname{expr}
$$

for $T E V I$ of [18] contains the logically independent factors $B \varepsilon \operatorname{expr} L$ and $A \varepsilon$ ingr $r_{\mathbf{L}}(B)$. That he does not intend

$$
[A B]: A \varepsilon \operatorname{ingr}_{\mathbf{L}}(B) . \supset . A \varepsilon \operatorname{expr}
$$

follows from $T E X X I I$ which contains the conjuncts $A$ \& expr $\mathrm{L}_{\mathrm{L}}$ and $A \varepsilon$ ingr $\mathrm{L}(B)$. That he does not intend this is also indicated by the complexity of TEVII.4. $C f$., also T2.4.6.

It is understandable that Leśniewski did not restrict ingr $L_{L}$ to expressions, for without this restriction we have just the usual notion of ingredient in a completely atomic Mereology. We used the more restrictive notion of ingredient to simplify our presentation of the T.E.'s.

$D f 1.9 \quad[A B]: A \varepsilon \operatorname{prcd}_{\mathbf{L}}(B) . \equiv . A \varepsilon \operatorname{prcd}(B)$ 
Leśniewski gives no commentary on his use of $\operatorname{prcd}_{\mathbf{L}}$. It is clear that strict precedence is intended, i.e.,

$$
[A B]: A \varepsilon \operatorname{prcd}_{\mathbf{L}}(B) . \supset . A \neq B
$$

for TEXXXII contains the conjunct

$$
A \varepsilon \operatorname{prcd}_{\mathbf{L}}(B) \cdot v \cdot A=B
$$

which would be redundant with weak precedence. At all other times predL is used in contexts like

$$
A \varepsilon \operatorname{vrb}_{\mathbf{L}} \cap \operatorname{prcd}_{\mathbf{L}}(B)
$$

or

$$
A \varepsilon \operatorname{expr}_{\mathbf{L}} \cap \operatorname{prcd}_{\mathbf{L}}(B)
$$

(Cf., TELX.3, XVI.3, XXV.4, XLVII.3, 4, 5, 6,IL.1) and so we cannot decide if he intends

$$
[A B]: A \varepsilon \operatorname{prcd} \mathbf{L} . \supset . A \varepsilon \operatorname{expr} . B \varepsilon \operatorname{expr}
$$

The interpretation of the rule does not depend on whether we consider precedence as restricted to expressions, or holding for arbitrary inscriptions. We have opted for the later.

After similar argumentation we define

$D f 1.10 \quad[A B]: A \varepsilon \operatorname{scd}_{\mathbf{L}}(B) . \equiv . A \varepsilon \operatorname{scd}(B)$

We make no comments about the remaining definitions as there is no reason to question their correctness.

$D f 1.11[A B]: A \varepsilon \operatorname{Uprcd}_{\mathbf{L}}(B) . \equiv . A \varepsilon \operatorname{Uprcd}_{(B)}$

$D f 1.12[A B]: A \varepsilon \operatorname{Uingr}_{\mathbf{L}}(B) . \equiv A \varepsilon \operatorname{Uvrb}(B)$

$D f 1.13[A B]: A \varepsilon 1$ ingr $_{\mathrm{L}}(B) . \equiv . A \varepsilon 1 \mathrm{vrb}(B)$

$D f 1.14[A B]: A \varepsilon 2$ ingr $_{\mathbf{L}}(B) \equiv A \varepsilon 2 \mathrm{vrb}(B)$

We now make the claim that Leśniewski's and our primitive terms are interdefinable in system MP, and hence our statement of the rule and his rest on the same logical basis. This claim is partly justified by the argumentation above, but mainly by intimate knowledge of [18]. The methods used above have followed, in spirit, Goodman's comments on constructional definitions [10].

2 Methodological Scheme. Each of Leśniewski's Terminological Explanations is in the form of an ontological definition:

(1) $[A \ldots]: A \varepsilon \Phi(\ldots) . \equiv \psi(A \ldots)$

where $\Phi$ is the defined term. $\psi(A \ldots)$ is a well formed formula built up from Leśniewski's primitive and previously defined terms. As such, this is not a formula in MP. But since in 1 we defined Leśniewski's primitives in system MP, there is a natural translation of (1) into a formula (2) in MP:

(2) $[A \ldots]: A \varepsilon \Phi_{\mathrm{L}}(\ldots) \equiv . \psi_{\mathrm{L}}(A \ldots)$ 
where the subscripted L's indicate that we are using the analogues of Leśniewski's terms in MP. Thus whenever Leśniewski writes

$$
A \varepsilon \operatorname{ingr}(B)
$$

in $[18]$ we shall write

$$
A \varepsilon \operatorname{ingr}_{\mathbf{L}}(\dot{B})
$$

as its natural translation into MP.

In Chapter $I V$ we defined what we wish to prove are terms equivalent to Leśniewski's. To do this we prove, in each of the following paragraphs, theorems of the form

(3) $[A \ldots]: A \varepsilon \Phi_{\mathrm{L}}(\ldots) . \equiv A \varepsilon \Phi_{\mathrm{R}}(\ldots)$

where $\Phi_{R}$ is a term defined in one of the T.E.'s in Chapter IV. These theorems establish that our terminological explanations are equivalent to those given by Leśniewski in [18]. Only when our terms are proved equivalent to Leśniewski's will it be clear that our statement of the rule of procedure for Protothetic is equivalent to that stated by Leśniewski in [18].

3 Concerning TEI (vrbi).

Df3 $[A]: A \varepsilon \operatorname{vrb}_{L} 1 . \equiv . A \varepsilon \operatorname{cnf}_{\mathbf{L}}\left(\operatorname{lingr}_{\mathbf{L}}(\mathfrak{H} \mathfrak{x})\right)$

$A$ is a word of the first kind (in the sense of Leśniewski). This definition is the exact analogue of Leśniewski's TEI. Since we have not defined "vrbl" in Chapter $I V$ we cannot prove a theorem of the desired form (3) without first defining "vrbl".

Df3.1 $[A]: A \varepsilon \operatorname{vrbl} . \equiv . A \varepsilon \operatorname{cnf}(1 \mathrm{vrb}(\mathfrak{A} \mathfrak{x}))$

Since our notion of equiformity differs from Leśniewski's we need several lemmas.

T5.3.1 $[A B]: A \varepsilon 1$ ingr $_{\mathbf{L}}(B)$..$A \varepsilon \mathrm{vrb}$

T5.3.2 $[A]: A \varepsilon \mathbf{v r b} . \supset \operatorname{cnf}(A) \circ \operatorname{cnf}_{\mathbf{L}}(A)$

$[D f 1.13, D 3, T 2.2 .9]$

T5.3.3 $[A]: A \varepsilon v v^{\prime} b_{L} \mid . \equiv . A \varepsilon v \operatorname{vrl}$

$[D f 3, D f 3.1, D f 1.13, T 5.3 .1, T 5.3 .2]$

4 Concerning TEII, III, IV (vrb2, vrb3, vrb4).

Df4.1 $[A]: A \varepsilon \operatorname{vrb}_{L} 2 . \equiv A \varepsilon \operatorname{cnf}_{\mathrm{L}}(4 \operatorname{ingr} \mathrm{L}(\mathfrak{A} \underline{x}))$

Df4.2 $[A]: A \varepsilon \operatorname{vrb}_{\mathrm{L}} 3 . \equiv . A \varepsilon \operatorname{cnf}_{\mathrm{L}}(5 \operatorname{ingr} \mathrm{L}(\mathfrak{A} \mathfrak{x}))$

Df4.3 $[A]: A \varepsilon \operatorname{vrb}_{\mathrm{L}} 4 . \equiv A \varepsilon \operatorname{cnf}_{\mathrm{L}}\left(\operatorname{Uingr}_{\mathrm{L}}(\mathfrak{H} \mathfrak{x})\right)$

These are Leśniewski's TEII, III, IV, adjusted to Sobociński's Axiom. In complete analogy with 3 we need the definitions

$D f 4.4 \quad[A]: A \varepsilon \operatorname{vrb} 2 . \equiv . A \varepsilon \operatorname{cnf}(4 \mathrm{vrb}(\mathfrak{A} \mathfrak{x}))$

Df4.5 $[A]: A \varepsilon \operatorname{vrb} 3 . \equiv . A \varepsilon \operatorname{cnf}(5 \operatorname{vrb}(\mathfrak{A r}))$

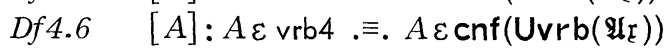

to prove the theorems

T5.4.1 $[A]: A \varepsilon v \operatorname{vrb}\llcorner 2$. $A \varepsilon \operatorname{vrb2}$ 
T5.4.2 $[A]: A \varepsilon \operatorname{vrb}^{3} 3 . \equiv . A \varepsilon \operatorname{vrb3}$

T5.4.3 $[A]: A \varepsilon \operatorname{vrb}_{\llcorner} 4$... $A \varepsilon \vee \operatorname{vrb} 4$

5 Concerning TEV (trm).

Df5 $[A]: A \varepsilon \operatorname{trm} \mathrm{L} . \equiv$. $A \varepsilon$ vrbL.

$A \varepsilon \sim\left[\right.$ prnt $\left._{L}\right]$.

$A \varepsilon N\left[v^{\prime} b_{L} 1\right]$.

$A \varepsilon N\left[v^{\prime} b_{L} 2\right]$.

$A \varepsilon N[\operatorname{vrbL} 3]$.

$A \varepsilon \sim\left[\mathrm{vrb}_{\mathrm{L}} 4\right]$

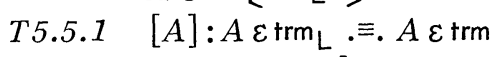

$[T E 1, D f 5, D f 1.1, D f 1.3, T 5.3 .3, T 5.4 .1, T 5.4 .2, T 5.4 .3]$

$D f 5$ is the exact analogue of Leśniewski's TEV. The theorem, which is of the desired form (3), proves that our TE1 has the same strength as Leśniewski's TEV.

6 Concerning TEVI (int).

$D f 6 \quad[A B]: A \varepsilon \operatorname{int}_{\mathbf{L}}(B) . \equiv$.

$B \varepsilon \operatorname{expr}$.

$A \varepsilon \mathrm{vrbL}$.

$A \varepsilon$ ingr $_{\mathbf{L}}(B)$.

$A \varepsilon N\left[1 \operatorname{lingr}_{\mathrm{L}}(B)\right]$.

$A \varepsilon N[\operatorname{Uingr}(B)]$

T5.6.1 $[A B]: A \varepsilon \mathbf{v r b}(B)$. $. A \varepsilon \mathbf{v r b}_{\mathbf{L}} . A \varepsilon \operatorname{ingr}_{\mathbf{L}}(B)$

$\operatorname{Hyp}(1) . \supset$.

2. $A \varepsilon v r b_{\mathrm{L}}$.

3. $A \varepsilon$ ingr $_{\mathbf{L}}(B)$.

$A \varepsilon v \mathrm{vrb}_{\mathrm{L}} . A \varepsilon \operatorname{ingr} \mathrm{L}(B)$

$[1, T 2.2 .9, D f 1.1]$

$[1, T 2.2 .7, D f 1.8]$

$[2,3]$

T5.6.2 $[A B]: A \varepsilon \operatorname{vrb}_{\mathrm{L}} . A \varepsilon \operatorname{ingr}_{\mathbf{L}}(B)$.ว. $A \varepsilon \operatorname{vrb}(B)$

$\operatorname{Hyp}(2) . \supset$.

3. $A \varepsilon \operatorname{vrb}(A)$.

$A \varepsilon \operatorname{vrb}(B)$

$[1, D f 1.1, D 2]$

$[2, D f 1.8,3]$

T5.6.3 $[A B]: A \varepsilon \operatorname{vrb}(B) . \equiv A \varepsilon \operatorname{vrb}_{\mathbf{L}} \cdot A \varepsilon \operatorname{ingr}_{\mathbf{L}}(B)$

T5.6.4 $[A B]: A \varepsilon \operatorname{int}_{\mathrm{L}}(B) . \equiv . A \varepsilon \operatorname{int}(B)$

$[T 5.6 .1, T 5.6 .2]$

$[D f 6, D 13, D f 1.2, D f 1.13, D f 1.14, T 5.6 .3]$

7 Concerning TEVII (Cmpl).

Df7 $\quad[A a]:: A \varepsilon \mathrm{Cmpl}_{\mathrm{L}}(A) . \equiv: . A \varepsilon \operatorname{expr}_{\mathrm{L}}:$.

$\left.[B]: B \varepsilon \mathbf{v r b}_{\mathrm{L}} . B \varepsilon \operatorname{ingr}_{\mathbf{L}}(A) . \supset .{ }_{\exists} C\right] . C \varepsilon a . B \varepsilon \operatorname{ingr}_{\mathbf{L}}(C):$.

$[B C D]: B \varepsilon a . C \varepsilon a . D \varepsilon \mathrm{vrb}_{\mathrm{L}} . D \varepsilon$ ingr $\mathrm{L}(B) . D \varepsilon \operatorname{ingr}_{\mathrm{L}}(C) . \supset . B=C$ :

$[B]: B \varepsilon a . \supset . B \varepsilon \operatorname{expr} \mathrm{L} \cap$ ingr $\mathbf{L}(A)$

$T 5.7 .1[a]: . \operatorname{disj}(a) . \equiv:[B C D]: B \varepsilon a . C \varepsilon a . D \varepsilon \mathbf{v r b}_{\mathrm{L}} . D \varepsilon \operatorname{ingr}_{\mathbf{L}}(B)$. $D \varepsilon$ ingr $_{\mathrm{L}}(C)$. $\supset . B=C$

$[D 11, T 5.6 .3]$

T5.7.2 $[A B a]: A \varepsilon \mathrm{KI}(a) . B \varepsilon a . \supset . B \varepsilon$ ingr $_{\mathrm{L}}(A)$

$[D 1, D f 1.8]$

T5.7.3 $[A a]: . A \varepsilon \mathrm{KI}(a) . a \subset \operatorname{expr} . \supset:[B]: B \varepsilon a$..$B \varepsilon \operatorname{expr}_{\mathrm{L}} \cap \operatorname{ingr}_{\mathrm{L}}(A)$ 
T5.7.4 $[A B a]: A \varepsilon \mathrm{KI}(a) . B \varepsilon \operatorname{vrb}_{\mathrm{L}} . B \varepsilon \operatorname{ingr}_{\mathrm{L}}(A) . \supset .\left[{ }_{\exists} C\right] C \varepsilon a \cdot B \varepsilon \operatorname{ingr}_{\mathrm{L}}(C)$ $\operatorname{Hyp}(3)$.つ.

$\left[{ }_{3} C\right]$.

$\left.\begin{array}{ll}\text { 4. } & C \varepsilon a \\ \text { 5. } & B \varepsilon \operatorname{vrb}(C) .\end{array}\right\}$

$[1, D 1, T 5.6 .3,2,3]$

$\left[{ }_{\exists} C\right] . C \varepsilon a . B \varepsilon$ ingr $\mathrm{L}(C)$

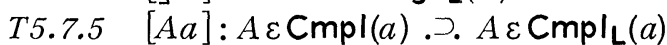

$[4,5, T 5.6 .3]$

[D12, Df7, Df1.2, T5.7.4, T5.7.1, T5.7.3]

T5.7.6 $[A a]: . A \varepsilon A:[B]: B \varepsilon \operatorname{vrb}_{\mathbf{L}} \cdot B \varepsilon \operatorname{ingr}_{\mathbf{L}}(A) . \supset .\left[{ }_{\exists} C\right] . C \varepsilon a$.

$B \varepsilon \operatorname{ingr}_{\mathbf{L}}(C):[B]: B \varepsilon a$.ว. $B \varepsilon \operatorname{expr}_{\mathbf{L}} \cap \operatorname{ingr}_{\mathbf{L}}(A): \supset . A \varepsilon \mathrm{KI}(a)$

Hyp(3) .Ј:

4. $[B]: B \varepsilon \operatorname{vrb}(A)$. . $\left[{ }_{\exists} C\right] . C \varepsilon a . B \varepsilon \operatorname{vrb}(C)$ :

$[2, T 5.6 .3]$

5. $[B C]: C \varepsilon a . B \varepsilon \operatorname{vrb}(C)$..$B \varepsilon \operatorname{vrb}(A)$ :

$[3, D f 1.8]$

$A \varepsilon \mathrm{KI}(a)$

T5.7.7 $[A a]: A \varepsilon \mathrm{Cmpl}_{\mathrm{L}}(a) . \equiv . A \varepsilon \mathrm{Cmpl}(a)$

$[D 1,1,4,5]$

[D12, Df7, T5.7.6, Df1.2, T5.7.1; T5.7.5]

8 Concerning TEVIII (qnif).

Df 8

$[A]: . A \varepsilon \operatorname{qntf}_{\mathrm{L}} \equiv$ :

$1 \operatorname{ingr}_{L}(A) \varepsilon \operatorname{vrb}_{L} 1$.

Uingr $_{L}(A) \varepsilon \operatorname{vrb}_{L} 2$.

$\left[{ }_{\exists} B\right] . B \varepsilon \operatorname{int}_{\mathbf{L}}(A)$ :

$[B]: B \varepsilon \operatorname{int}_{\mathrm{L}}(A)$.つ. $B \varepsilon \operatorname{trm}_{\mathrm{L}}$ :

$[B C]: B \varepsilon \operatorname{int}_{\mathrm{L}}(A) . C \varepsilon \operatorname{int}_{\mathrm{L}}(A) . B \varepsilon \operatorname{cnf}_{\mathrm{L}}(C) . \supset . B=C$

T5.8.1 $[A]: 1 \operatorname{ingr}_{\mathbf{L}}(A) \varepsilon \operatorname{vrb}_{\mathcal{L}} 1 . \equiv . \operatorname{vrbb}(A) \varepsilon \operatorname{enf}(1 \operatorname{vrb}(\mathfrak{A} \mathfrak{x}))$

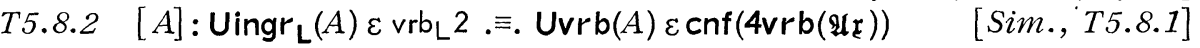

T5.8.3 $[A]: \operatorname{lnt}(A) \varepsilon \mathrm{Cmpl}(\operatorname{trm} \cap \operatorname{int}(A)) . \supset .\left[{ }_{\exists} B\right] . B \varepsilon \operatorname{int}_{\mathrm{L}}(A)$

$[D 14, T 2.3 .4, T 5.6 .4]$

$T 5.8 .4[A B]: \operatorname{lnt}(A) \varepsilon \mathrm{Cmpl}(\operatorname{trm} \cap \operatorname{int}(A)) . B \varepsilon \operatorname{int}_{\mathrm{L}}(A)$.Ј. $B \varepsilon \operatorname{trm}_{\mathrm{L}}$ $\operatorname{Hyp}(2) . \supset$.

3. $B \varepsilon \operatorname{int}(A)$.

$[2, T 5.6 .4]$

4. $B \varepsilon \mathrm{vrb}$.

$[3, D 13, T 2.2 .9]$

5. $B \varepsilon \operatorname{vrb}(\operatorname{Int}(A))$.

$\left[{ }_{\exists} C\right]$.

6. $\quad C \varepsilon \operatorname{trm}$.

7. $\quad B \varepsilon \operatorname{vrb}(C)$.

$[1,5, D 12, D 1]$

8. $\quad C \varepsilon \mathrm{vrb}$.

$[6, T E 1]$

9. $B=C$.

$[7,8, T 2.2 .10]$

$B \varepsilon \operatorname{trm} \mathrm{L}$

$[6,9, T 5.5 .1]$

T5.8.5 $[A B C]: A \varepsilon$ non-rep. $B \varepsilon \operatorname{int}_{\mathbf{L}}(A) . C \varepsilon \operatorname{int}_{\mathbf{L}}(A) . B \varepsilon \operatorname{cnf}_{\mathbf{L}}(C) . \supset . B=C$ Hyp(4) .つ.

5. $B \varepsilon \operatorname{vrb}(A)$.

$[2, T 5.6 .4, D 13]$

6. $C \varepsilon \operatorname{vrb}(A)$.

$[3, T 5.6 .4, D 13]$

7. $B \varepsilon \operatorname{cnf}(C)$.

$[4, D f 1.6]$

$B=C$

$[D 19,1,5,6,7]$

T5.8.6 $[A]: A$ \&qntf .ว. $A \varepsilon$ qntf $_{L}$

[TE2, Df8, T5.8.1-5] 


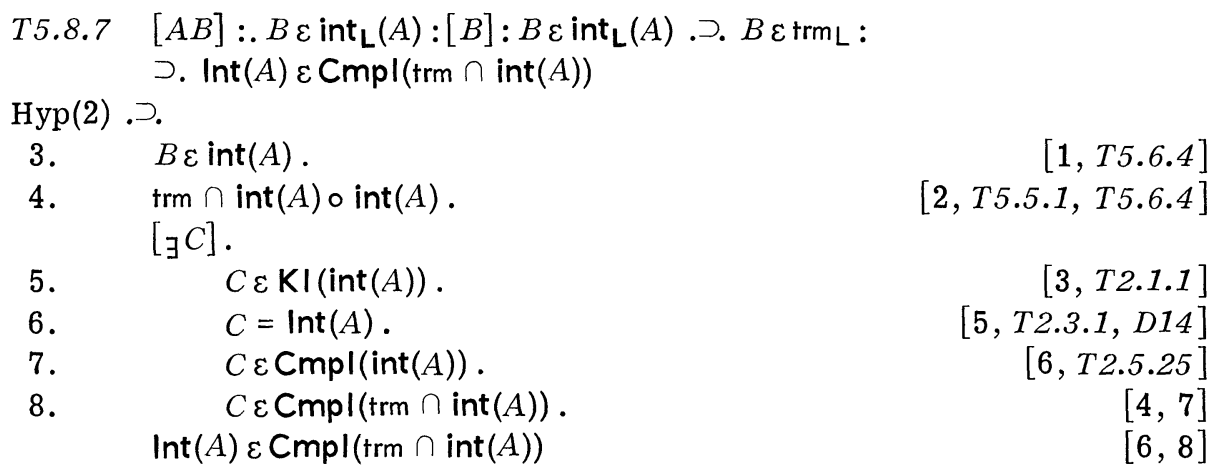

In Leśniewski's presentation TEVIII (qntf) contains the clause

$$
[B C]: B \varepsilon \operatorname{int}_{\mathbf{L}}(A) . C \varepsilon \operatorname{int}_{\mathbf{L}}(A) . B \varepsilon \operatorname{cnf}_{\mathbf{L}}(C) . \supset . B=C
$$

which is equivalent to

$$
\operatorname{lnt}(A) \varepsilon \text { non-rep }
$$

In $T E 2$ we used

$$
A \varepsilon \text { non-rep }
$$

This is possible since a generalization begins and ends with (left and right respectively) corners, which are not terms and hence not equiform to any word in $\operatorname{lnt}(A)$.

From $D 13$ we obtain at once

$[A B]: B \varepsilon \operatorname{vrb}(A) . A \varepsilon \operatorname{expr} . \supset: B \varepsilon \operatorname{int}(A)$.v. $B \varepsilon 1 \mathrm{vrb}(A)$.v. $B \varepsilon \operatorname{Uvrb}(A)$

Hence when we take two words in (but not necessarily interior to) $A$ we obtain the following nine possibilities:

$$
\begin{aligned}
& \text { I. } B \varepsilon \operatorname{int}(A) . C \varepsilon \operatorname{int}(A) \\
& \text { II. } B \varepsilon \operatorname{int}(A) . C \varepsilon \operatorname{ivrb}(A) \\
& \text { III. } B \varepsilon \operatorname{int}(A) . C \varepsilon \operatorname{Urrb}(A) \\
& \text { IV. } B \varepsilon \operatorname{lvrb}(A) . C \varepsilon \operatorname{int}(A) \\
& \text { V. } B \varepsilon \operatorname{lvrb}(A) . C \varepsilon \operatorname{1vrb}(A) \\
& \text { VI. } B \varepsilon 1 \mathrm{vrb}(A) . C \varepsilon \operatorname{Urrb}(A) \\
& \text { VII. } B \varepsilon \operatorname{Urrb}(A) . C \varepsilon \operatorname{int}(A) \\
& \text { VIII. } B \varepsilon \operatorname{Uvrb}(A) . C \varepsilon 1 \mathrm{vrb}(A) \\
& \text { IX. } B \varepsilon \operatorname{Urrb}(A) . C \varepsilon \operatorname{Urrb}(A)
\end{aligned}
$$

We wish to establish theorems of the form $[A B C]: A \varepsilon \operatorname{qntf}_{\mathrm{L}} . B \varepsilon \operatorname{cnf}(C) . Y . \supset . B=C$

where $Y$ is one of I-IX. Taken together these yield

$$
[A]: A \varepsilon \text { qntf }_{L} . \supset . A \varepsilon \text { non-rep }
$$

Of the nine cases IV, VII, VIII can be omitted by symmetry with II, III, VI respectively. Case VI is impossible since $L$ and $\lrcorner$ are not equiform. 
Cases V, IX are just T2.2.30, T2.2.31. So there remain three cases to consider.

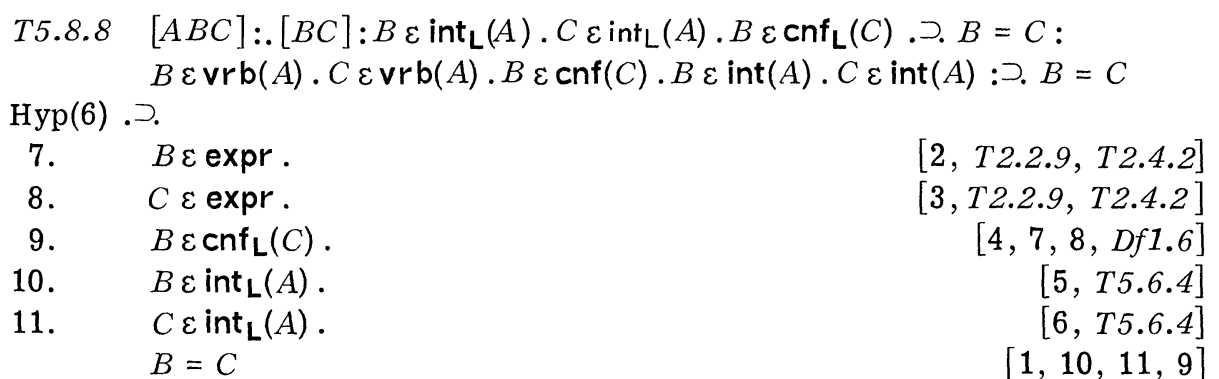

T5.8.9 $[A B C]: .1 \operatorname{ingr}_{\mathrm{L}}(A) \varepsilon \operatorname{vrb}_{\mathrm{L}} 1:[B]: B \varepsilon \operatorname{int}_{\mathrm{L}}(A) . \supset . B \varepsilon \operatorname{trm}_{\mathrm{L}}: B \varepsilon \operatorname{cnf}(C)$. $B \varepsilon \operatorname{int}(A) . C \varepsilon \operatorname{lvrb}(A): \supset . B=C$

$\operatorname{Hyp}(5)$. .

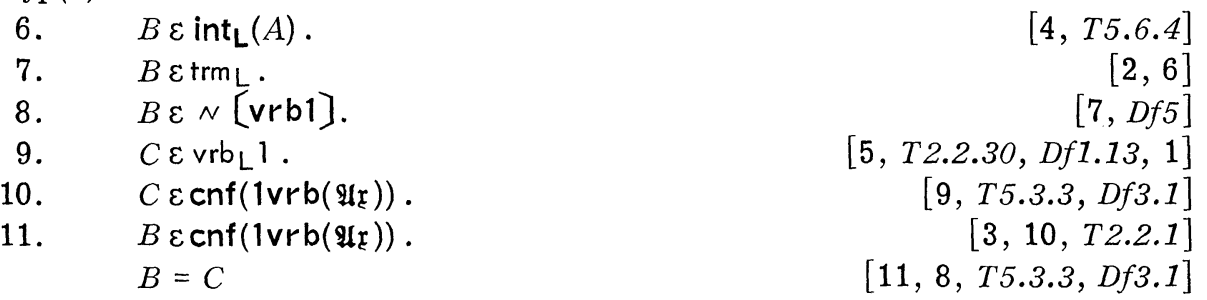

T5.8.10 $[A B C]:$. Uingr $(A) \varepsilon \operatorname{vrb}_{\mathrm{L}} 2:[B]: B \varepsilon \operatorname{int}_{\mathrm{L}}(A) . \supset . B \varepsilon \operatorname{trm}_{\mathrm{L}}$ :

$B \varepsilon \operatorname{cnf}(C) . B \varepsilon \operatorname{int}(A) . C \varepsilon \operatorname{Urb}(A): \supset . B=C \quad$ [Sim., T5.8.9]

T5.8.11 $[A B C]: B \varepsilon 1 \mathrm{vrb}(A) . C \varepsilon \operatorname{Urrb}(A) . B \varepsilon \operatorname{cnf}(C) .1$ ingr $_{\mathrm{L}}(A) \varepsilon \operatorname{vrb}_{L} 1$. $\operatorname{Uingr}_{\mathbf{L}}(A) \varepsilon \operatorname{vrb}_{\mathrm{L}} 2 . \supset . B=C$

$\operatorname{Hyp}(5)$.Ј.

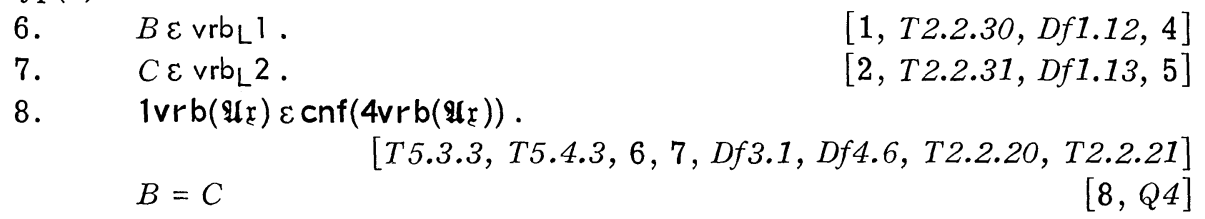

Finally we are able to state the desired theorem

T5.8.11.1 [A]:.A $A \operatorname{qntf}_{L} . B \varepsilon \operatorname{vrb}(A) . C \varepsilon \operatorname{vrb}(A) . B \varepsilon \operatorname{cnf}(C) . \supset . B=C$ $\mathrm{Hyp}(4) . \supset$ :

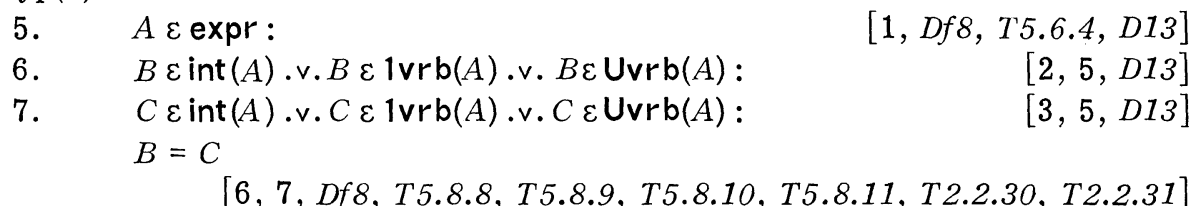

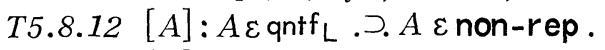

$[T 5.8 .11 .1, D 19]$

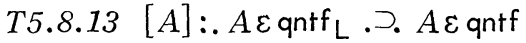

$[D f 8, T E 2, T 5.8 .1, T 5.8 .2, T 5.8 .7, T 5.8 .12]$

T5.8.14 $[A]: A \varepsilon \operatorname{qntf}_{L} \equiv . A \varepsilon q \operatorname{qntf}$

$[T 5.8 .6, T 5.8 .13]$

We now continue in this fashion introducing definitions of Leśniewski's terms into our system and then proving that our terms are equivalent to 
his. In this way we obtain theorems which show that each of the terms used in the statement of the rule of Protothetic that we gave is equivalent to a term used in Leśniewski's statement. Hence the two statements of the rule are equivalent in the sense that they allow precisely the same deductions. These proofs, the details of which are too long to include here, can be found in $[46]$.

\section{CONCLUSION}

As we have stressed earlier the most important part of this work is the first, particularly Chapter II. There we presented and developed a system $M$ of metalogic. Our aim in constructing this system was twofold. We wished it to be adequate for the statement of procedural rules of formalized systems, and wished it also to be adequate for conducting some metalogical investigations. Our claim is that, with varying degrees of success, both of these aims have been accomplished.

Clearly a proof that we can formulate rules for any formalized system in $M$, or even in extensions of $M$ like $M P$, is out of the question at this time. To do so would require a precise notion of "rule of procedure" which is, to our knowledge, not available at this time. Probably if such a definition did exist we could easily establish whether all such rules were expressible in $\mathrm{M}$. Since such a proof was beyond our grasp we adopted the following technique.

Protothetic is a system with rules which are complicated to state precisely, and strong enough so that they encompass the rules of most logical theories. Thus there can be little doubt that the statement of the rule of Protothetic is a challenging test of the first part of our claim.

For one familiar with Leśniewski's systems it should be clear that the rule of Ontology can be presented analogously to the rule of Protothetic. Moreover, using the results of Chapter IV we could simplify the statement of the rule of Ontology given by Leśniewski in [19].

It seems almost banal to remark that system $M$ is sufficient to state the rules for propositional calculi. If the only rules are substitution and detachment then the statement of the rule is trivial; the propositional constants all occur in the axioms and so we can just specify all symbols not equiform to one of those to be variables. If the calculus contains definitions then more care is required. Cf. Leśniewski [20].

By modifying the techniques of Chapter III, IV we can present the rules for functional calculi of any order and for set theories. For Gentzen like systems it is not so obvious that we can use similar techniques. The main difficulties are the linearization of the language and allowing for expressions of arbitrary (even infinite) length. This would require us to drop the axiom concerning the finiteness of inscriptions.

In regard to the other half of our claim we have shown in Chapter $I V$ that system $M$, or really MP, is adequate for some metalogical investigations, viz., proof of the equivalence of different statements of rules. A most interesting question is what type of metalogical investigations can actually be carried out in system $\mathbf{M}$. Probably consistency and completeness 
of propositional systems could be carried out completely in $M$, but it seems highly doubtful that incompleteness results could be established within $M$ without the use of variables of higher types and new axioms involving these types.

\section{FOOTNOTES}

1. Cf. TE51, Chapter IV, 5.

2. The following definition, due to Sobocinski [47], is more perspicuous:

$$
[p q]: . p . q . \equiv:[f]: f(p q) . \equiv . f(11)
$$

3. In systems of Protothetic based on implication definitions must be stated either as pairs of implications:

$$
\begin{aligned}
& \alpha \supset \beta \\
& \beta \supset \alpha
\end{aligned}
$$

or in the complicated form

$$
\alpha \supset \beta . \supset: \beta \supset \alpha . \supset p:: \supset p
$$

where both the definiens $\alpha$ and definiendum $\beta$ are free in $p, c f$. Lejewski [50].

4. The symbolism used here is that of Leśniewski. Once the key to the "wheel and spoke" notation is explained it is easy to remember. The symbol $\phi$ corresponds to the truth table

\begin{tabular}{r|rr} 
& 0 & 1 \\
\hline 0 & 1 & 0 \\
$*_{1}$ & 0 & 1
\end{tabular}

i.e., to equivalence. In general a spoke at the top (right, bottom, left) means a 1 in the $(0-0)$-place (resp $(0-1),(1-1),(1-0)$-place) of the truth table. Thus $P$ is conjunction for it corresponds to

\begin{tabular}{r|ll} 
& 0 & 1 \\
\hline 0 & 0 & 0 \\
$*_{1}$ & 0 & 1
\end{tabular}

It should be understood that this symbolism, while very convenient, is not mandatory. Protothetic is formulated in a way which leaves all possible choices of symbolism up to the individual.

5. Interchanging functors in the same category need not preserve truth, and generally it does not.

6. One reason for rejecting incomplete symbols is that the rule of substitution has never been strictly formulated using them.

7. Just like 'man' is a growing name. Every time a baby is born the name 'man' denotes one more object.

8. Whether a parenthesis is called left or right is a matter of having a familiar looking notation. We could just as well write ' $)(p$ ' as ' $(p)$ '. No confusion can arise as the introductory thesis for a new pair of parentheses determines which of the two is called left. 
9. We have used different enumerations on the axioms to indicate that they are of different natures. The $P$ axioms concern the primitive terms of MP. The $Q$ axioms are statements about the axiom $\mathfrak{A r}$ of Protothetic. They are of empirical origin, but must be introduced as axioms into system MP.

10. The Terminological Explanations stated in this Chapter are different than those in Leśniewski [18]. They will be proved equivalent in Chapter $V$.

11. By the $n$-th conjunct of a T.E. we mean the $n$-th conjunct on the right hand side of the main equivalence of the T.E. More specifically TEMn will denote the $n$-th conjunct of TEM. The conjuncts of the T.E.'s are independent. This claim implies that the 1 st, 4 th, 5 th and Uvrb of $\mathfrak{A r}$ are not equiform.

\section{BIBLIOGRAPHY}

Items [1]-[44] were given in Part I of this paper. See Notre Dame Journal of Formal Logic, vol. XIII (1972), pp. 1-33.

45. Bar-Hillel, Yehoshua, "On Syntactical Categories," The Journal of Symbolic Logic, vol. 15 (1950), pp. 1-16.

46. Rickey, V. Frederick, An Axiomatic Theory of Syntax, $\mathrm{PhD}$ thesis, University of Notre Dame, Notre Dame, Indiana (August 1968).

47. Sobociński, Bolesław, "An Investigation of Protothetic," Cahiers de l'Institut d'Etudes Polonaises en Belgique, No. 5, Brussels (1949). Reprinted in [25], pp. 201-206. Unfortunately the reprint omits the "Introduction (pp. 7-27) of unusual expository and bibiliographical interest, giving an account of the illfated Polish periodical Collectanea Logica." See the review by Church, The Journal of Symbolic Logic, vol. 15 (1950), p. 64.

48. Sobociński, Bolesław, "On the single axioms of Protothetic," Notre Dame Journal of Formal Logic, vol. I (1960), pp. 52-73, vol. II (1961), pp. 111-126, 129-148. (To be continued.)

49. Tarski, Alfred, “O wyrazie pierwotnym logistyki," Przeglad Filozoficzny, vol. 26 (1923), pp. 68-69. Translated in [44], pp. 1-23.

50. Lejewski, C., “On Implicational Definitions," Studia Logica, vol. 8 (1958), pp. 189-211.

Seminar in Symbolic Logic

University of Notre Dame

and

Bowling Green State University

Bowling Green, Ohio 